4 Zhiyuan Chen,,${ }^{\dagger++}$ Lan Yang,${ }^{\dagger,+}$ Yu Huang, ${ }^{\dagger}$ Peter Spencer, ${ }^{\dagger}$ Weiwei Zheng, ${ }^{\dagger}$ Ying Zhou, ${ }^{\dagger}$

5 Songhui Jiang, ${ }^{\dagger}$ Weimin Ye, ${ }^{\S}$ Yuxin Zheng,,$\|$ Weidong Qu $*, \dagger$

\section{Carcinogenic risk of $N$-Nitrosamines in Shanghai Drinking Water: Indications for the Use of Ozone Pretreatment}

${ }^{\dagger}$ Center for Water and Health, Key Laboratory of Health Technology Assessment, National Health Commission of the People's Republic of China, Key Laboratory of the Public Health Safety, Ministry of Education, Department of Environmental Health, School of Public Health, Fudan University, Shanghai, 200032, China

$\star$ Oregon Institute of Occupational Health Sciences, and Department of Neurology, School of Medicine, Oregon Health \& Science University, Portland, Oregon, 97239, United States

\footnotetext{
${ }^{\S}$ Department of Medical Epidemiology and Biostatistics, Karolinska Institutet, Stockholm,
} 17177 , Sweden

" School of Public Health, Qingdao University, 38 Dengzhou Road, Qingdao, 266021, China 9

+ These authors contributed equally to this work.

*Corresponding author: Weidong Qu, Key Laboratory of the Public Health Safety, Ministry of Education, Department of Environmental Health, School of Public Health, Fudan University. Phone: 86-21-54237203; fax: 86-21-64045165; e-mail: wdqu@ fudan.edu.cn.

Running title: Cancer risk exposed to $N$-Nitrosamines in drinking water 

potential competing financial interests.

30

Table S1-19 please see page S3 to page S9

Figure S1-3 please see page $S 20$ to page $S 22$ 
Table S1. Compound name, retention time, concentration range, regression coefficient $\left(\mathrm{R}^{2}\right)$, recovery, precision, limit of detection (LOD), and internal standards (IS) of all target $N$-Nitrosamines.

\begin{tabular}{|c|c|c|c|c|c|c|c|c|c|}
\hline \multirow[t]{2}{*}{$\operatorname{compound}^{\mathrm{a}}$} & \multirow{2}{*}{$\begin{array}{l}\text { retention time } \\
\text { (min) }\end{array}$} & \multirow{2}{*}{$\begin{array}{c}\text { range } \\
(\mu \mathrm{g} / \mathrm{mL})\end{array}$} & \multirow[t]{2}{*}{$\mathbf{R}^{2}$} & \multicolumn{2}{|c|}{ recovery $(\%), n=6$} & \multicolumn{2}{|c|}{ precision $\left(\% \operatorname{RSD}^{b}\right), n=6$} & \multirow{2}{*}{$\begin{array}{c}\text { LOD } \\
(n g / L)\end{array}$} & \multirow[t]{2}{*}{ IS } \\
\hline & & & & $10 n g / L$ & $100 \mathrm{ng} / \mathrm{L}$ & $10 n g / L$ & $100 \mathrm{ng} / \mathrm{L}$ & & \\
\hline NDMA & 6.01 & $0.01-1$ & 0.9996 & 100.64 & 91.30 & 6.33 & 14.24 & 2.00 & Naphthalene- $\mathrm{d}_{8}$ \\
\hline NMEA & 7.56 & $0.01-1$ & 0.9995 & 94.26 & 99.44 & 11.63 & 13.89 & 3.45 & Naphthalene- $\mathrm{d}_{8}$ \\
\hline NDEA & 8.93 & $0.01-1$ & 0.9997 & 101.10 & 86.01 & 12.98 & 12.80 & 4.12 & Naphthalene- $\mathrm{d}_{8}$ \\
\hline NPYR & 12.38 & $0.01-1$ & 0.9985 & 100.71 & 90.85 & 8.40 & 13.04 & 2.66 & Naphthalene- $\mathrm{d}_{8}$ \\
\hline NMOR & 12.52 & $0.01-1$ & 0.9923 & 100.92 & 89.51 & 7.96 & 12.69 & 2.52 & Naphthalene- $\mathrm{d}_{8}$ \\
\hline NPIP & 13.28 & $0.01-1$ & 0.9973 & 100.29 & 93.80 & 3.34 & 12.88 & 1.05 & Naphthalene- $\mathrm{d}_{8}$ \\
\hline NDPA & 13.33 & $0.01-1$ & 0.9941 & 94.26 & 89.89 & 8.18 & 9.87 & 2.42 & Naphthalene- $\mathrm{d}_{8}$ \\
\hline NDBA & 16.28 & $0.01-1$ & 0.9947 & 88.87 & 85.28 & 7.92 & 14.86 & 2.21 & Naphthalene- $\mathrm{d}_{8}$ \\
\hline
\end{tabular}

a The full compound name: NDMA, $N$-nitrosodimethylamine; NMEA, $N$-nitrosomethylethylamine; NDEA, $N$-nitrosodiethylamine; NPYR, $N$-nitrosopyrrolidine; NMOR, $N$ nitrosomorpholine; NPIP, $N$-nitrosopiperidine; NDPA, $N$-nitrosodipropylamine; NDBA, $N$-nitrosodibutylamine. ${ }^{\mathrm{b}}$ Relative standard deviation. 
Table S2. Parameters values used for risk calculations in the central tendency exposre (CTE) and reasonable maximum exposure (RME) scenario.

\begin{tabular}{|c|c|c|c|c|c|c|c|c|c|c|c|c|c|c|c|}
\hline \multirow[t]{2}{*}{$\begin{array}{l}\text { Age Group }{ }^{\mathrm{a}} \\
\quad \text { (year) }\end{array}$} & \multicolumn{2}{|c|}{$\begin{array}{c}\text { IR }^{\mathbf{b}} \\
(L / \text { day }) \\
\end{array}$} & \multirow[t]{2}{*}{$\begin{array}{c}\operatorname{InhR}^{b} \\
\left(m^{3} / \text { day }\right)\end{array}$} & \multirow[t]{2}{*}{$\begin{array}{c}\text { EF } \\
\text { (day/year) }\end{array}$} & \multirow[t]{2}{*}{$\begin{array}{c}E^{c} \\
\text { (year) }\end{array}$} & \multirow[t]{2}{*}{$\begin{array}{c}E^{\mathrm{d}} \\
\text { (events/day) }\end{array}$} & \multicolumn{2}{|c|}{$\begin{array}{c}\mathbf{E T}^{\mathbf{b}} \\
(\mathrm{min} / \mathrm{day}) \\
\end{array}$} & \multicolumn{2}{|c|}{$\begin{array}{c}\mathbf{t}_{\text {event }}{ }^{b} \\
\text { (min/event) } \\
\end{array}$} & \multicolumn{2}{|c|}{$\begin{array}{c}\mathbf{S A}^{\mathbf{b}} \\
\left(\mathrm{cm}^{2}\right) \\
\end{array}$} & \multirow[t]{2}{*}{$\begin{array}{l}\mathrm{BW}^{\mathrm{b}} \\
(\mathrm{kg})\end{array}$} & \multirow[t]{2}{*}{$\begin{array}{c}\text { LT }(\mathrm{AT})^{\mathrm{c}} \\
(\text { days })\end{array}$} & \multirow[t]{2}{*}{ ADAF } \\
\hline & CTE & RME & & & & & CTE & RME & $\overline{\text { CTE }}$ & RME & CTE & RME & & & \\
\hline Birth to 0.25 & 0.182 & 0.6 & 3.7 & 365 & 0.25 & 1 & 5 & 16 & 5 & 16 & 3400 & 4300 & 6.4 & 91.25 & 10 \\
\hline 0.25 to 0.5 & 0.345 & 1.2 & 4.7 & 365 & 0.25 & 1 & 7 & 16 & 7 & 16 & 4000 & 4800 & 7.9 & 91.25 & 10 \\
\hline 0.5 to 0.75 & 0.592 & 1.625 & 5.4 & 365 & 0.25 & 1 & 8 & 19 & 8 & 19 & 4500 & 5200 & 9.1 & 91.25 & 10 \\
\hline 0.75 to 1 & 0.813 & 2.25 & 5.9 & 365 & 0.25 & 1 & 8 & 20 & 8 & 20 & 4700 & 5400 & 9.8 & 91.25 & 10 \\
\hline 1 to 2 & 0.911 & 2.125 & 5.7 & 365 & 1 & 1 & 8 & 19 & 8 & 19 & 5200 & 6100 & 11.2 & 365 & 10 \\
\hline 2 to 3 & 0.809 & 1.675 & 6.3 & 365 & 1 & 1 & 9 & 19 & 9 & 19 & 6100 & 7100 & 13.5 & 365 & 10 \\
\hline 3 to 4 & 0.863 & 1.75 & 8.0 & 365 & 1 & 1 & 9 & 19 & 9 & 19 & 6800 & 7900 & 15.6 & 365 & 3 \\
\hline 4 to 5 & 0.851 & 1.75 & 8.4 & 365 & 1 & 1 & 9 & 19 & 9 & 19 & 7400 & 8800 & 17.7 & 365 & 3 \\
\hline 5 to 6 & 0.861 & 1.75 & 8.8 & 365 & 1 & 1 & 9 & 18 & 9 & 18 & 8000 & 9500 & 19.6 & 365 & 3 \\
\hline 6 to 9 & 1.186 & 2.15 & 10.1 & 365 & 3 & 1 & 9 & 20 & 9 & 20 & 9900 & 12500 & 26.5 & 1095 & 3 \\
\hline 9 to 12 & 1.28 & 2.3 & 13.2 & 365 & 3 & 1 & 10 & 22 & 10 & 22 & 12300 & 15800 & 36.8 & 1095 & 3 \\
\hline 12 to 15 & 1.383 & 2.7 & 13.5 & 365 & 3 & 1 & 12 & 28 & 12 & 28 & 14600 & 17900 & 47.3 & 1095 & 3 \\
\hline 15 to 18 & 1.414 & 3.254 & 14 & 365 & 3 & 1 & 10 & 26 & 10 & 26 & 16100 & 19000 & 54.8 & 1095 & 3 \\
\hline Adults & 1.85 & 5.2 & 15.7 & 365 & 76.34 & 1 & 7 & 18 & 7 & 18 & 16000 & 19000 & 60.6 & 27864.1 & 1 \\
\hline 18 to 45 & 1.875 & 5.25 & 16 & 365 & 27 & 1 & 8 & 19 & 8 & 19 & 16000 & 19000 & 60.1 & 9855 & 1 \\
\hline 45 to 60 & 1.9 & 5.35 & 16 & 365 & 15 & 1 & 7 & 18 & 7 & 18 & 16000 & 19000 & 62.4 & 5475 & 1 \\
\hline 60 to 80 & 1.8 & 4.85 & 13.7 & 365 & 20 & 1 & 6 & 15 & 6 & 15 & 16000 & 19000 & 59.4 & 7300 & 1 \\
\hline$\geq 80$ & 1.525 & 4.5 & 12 & 365 & 20 & 1 & 5 & 16 & 5 & 16 & 15000 & 18000 & 54.3 & 7300 & 1 \\
\hline
\end{tabular}

${ }^{a}$ Chosen age intervals are recommended by the Exposure Factors Handbook of Chinese Population (0-5 years, 2016) (6-17 years, 2016) (adults, 2013). ${ }^{1-3}$

${ }^{\mathrm{b}} \mathrm{IR}$, ingestion rate; InhR, inhalation rate; ET, exposure time; $\mathrm{t}_{\text {event, }}$ event duration; SA, skin surface area; BW, body weight. Values of these parameters are derived from the Exposure Factors Handbook of Chinese Population.

${ }^{c}$ ED, exposure duration; LT, lifetime. Values of these parameters are derived from China Statistical Yearbook (2015). ${ }^{4}$

${ }^{\mathrm{d}} \mathrm{EV}$, event frequency. Value of EV is recommended by Exposure Factors Handbook from U.S. EPA. ${ }^{5}$ 
Table S3. Chemical-specific factors ${ }^{\text {a }}$, cancer classification and guideline values of patterns of $N$-Nitrosamines.

\begin{tabular}{|c|c|c|c|c|c|c|}
\hline Factors & NDEA & NDMA & NDPA & NMEA & NPYR & Data Source \\
\hline $\mathrm{K}_{\mathrm{p}}(\mathrm{cm} / \mathrm{hr})$ & $1.20 \mathrm{E}-03$ & $2.70 \mathrm{E}-04$ & $2.80 \mathrm{E}-03$ & $5.41 \mathrm{E}-04$ & $3.27 \mathrm{E}-04$ & U.S. EPA \\
\hline $\operatorname{HLC}\left(a \mathrm{tm} \cdot \mathrm{m}^{3} / \mathrm{mol}\right)$ & $6.55 \mathrm{E}-06\left(37^{\circ} \mathrm{C}\right)$ & $1.08 \mathrm{E}-06\left(20^{\circ} \mathrm{C}\right)$ & $5.38 \mathrm{E}-06\left(37^{\circ} \mathrm{C}\right)$ & $1.44 \mathrm{E}-06\left(20^{\circ} \mathrm{C}\right)$ & $4.89 \mathrm{E}-08\left(37^{\circ} \mathrm{C}\right)$ & HSBS \\
\hline FA (unitless) & 1 & 1 & 1 & 1 & 1 & U.S. EPA \\
\hline$\tau_{\text {event }}(\mathrm{hr} /$ event $)$ & $3.0 \mathrm{E}-01$ & $2.5 \mathrm{E}-01$ & $5.4 \mathrm{E}-01$ & 3.3E-01 & $3.8 \mathrm{E}-01$ & U.S. EPA \\
\hline $\mathrm{SF}(m \mathrm{~g} / \mathrm{kg} / \text { day })^{-1}$ & $1.5 \mathrm{E}+02$ & $5.1 \mathrm{E}+01$ & $7.0 \mathrm{E}+00$ & $2.2 \mathrm{E}+01$ & $2.1 \mathrm{E}+00$ & IRIS \\
\hline $\operatorname{IUR}\left(\mu \mathrm{g} / \mathrm{m}^{3}\right)^{-1}$ & 4.3E-02 & $1.4 \mathrm{E}-02$ & $2.0 \mathrm{E}-03$ & $6.3 \mathrm{E}-03$ & $6.1 \mathrm{E}-04$ & IRIS, OEHHA \\
\hline \multirow[t]{2}{*}{ Cancer Classification } & B2 & $\mathrm{B} 2$ & B2 & B2 & $\mathrm{B} 2$ & IRIS \\
\hline & $2 \mathrm{~A}$ & $2 \mathrm{~A}$ & $2 \mathrm{~B}$ & $2 \mathrm{~B}$ & $2 \mathrm{~B}$ & IARC \\
\hline \multicolumn{7}{|l|}{ Guideline Values (ng/L) } \\
\hline \multicolumn{7}{|l|}{ U.S. California, 2013} \\
\hline notification level & 10 & 10 & 10 & - & - & \\
\hline response level & 100 & 300 & 500 & - & - & \\
\hline Canada, 2010 & - & 40 & - & - & - & \\
\hline Australia, 2013 & - & 100 & - & - & - & \\
\hline WHO, 2006 & - & 100 & - & - & - & \\
\hline
\end{tabular}

${ }^{a} K_{\mathrm{p}}$ : Permeability coefficient, data of NDEA, NDMA and NDPA from Exposure Factors Handbook (2011), data of NMEA and NPYR from the formula Log $\mathrm{k}_{\mathrm{p}}=-$

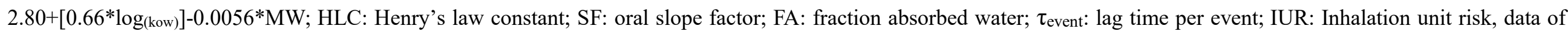
NDPA and NMEA from The Office of Environmental Health Hazard Assessment (OEHHA) (2009). B2: Probable human carcinogen defined by IRIS (2002); 2A: Probably carcinogenic to humans defined by International Agency for Research on Cancer (IARC) (2013); 2B: Possibly carcinogenic to humans defined by IARC (2013). 
Table S4. Parameter values used for the calculation of disease burden. ${ }^{6-9}$

\begin{tabular}{|c|c|c|c|c|c|c|c|c|c|c|c|}
\hline \multirow[t]{2}{*}{ Parameter } & \multirow{2}{*}{$\begin{array}{l}\text { Age-weighting } \\
\text { Factor } \mathrm{C}\end{array}$} & \multirow{2}{*}{$\begin{array}{l}\text { Age-weighting } \\
\text { Function Parameter } \beta\end{array}$} & \multirow{2}{*}{$\begin{array}{l}\text { Annual } \\
\text { Discount Rate } \mathbf{r}\end{array}$} & \multirow{2}{*}{$\begin{array}{l}\text { Disability } \\
\text { Weights D }\end{array}$} & \multicolumn{5}{|c|}{ Duration of Disability (year) } & \multicolumn{2}{|c|}{ Life Expectancy } \\
\hline & & & & & $0-4$ year & 5-14 year & $15-44$ year & $45-59$ year & 60 year- & Male & Female \\
\hline Values & 0.1658 & 0.04 & $3 \%$ & 0.78 & 0.00 & 0.00 & 4.56 & 3.68 & 2.78 & 71.5 & 78.5 \\
\hline
\end{tabular}

Table S5. Regional screening levels (SL) $(\mu \mathrm{g} / \mathrm{L})$ corresponding to a $10^{-4}$ risk level for $N$-Nitrosamines. ${ }^{10}$

\begin{tabular}{llllll}
\hline $\boldsymbol{N}$-Nitrosamines & CAS No. & Ingestion SL & Dermal SL & Inhalation SL & Carcinogenic SL \\
\hline NDEA & $55-18-5$ & $1.7 \mathrm{E}-02$ & $1.7 \mathrm{E}+00$ & - & $1.7 \mathrm{E}-02$ \\
NDMA & $62-75-9$ & $4.9 \mathrm{E}-02$ & $2.0 \mathrm{E}+01$ & $1.4 \mathrm{E}-02$ & $1.1 \mathrm{E}-02$ \\
NDPA & $621-64-7$ & $1.1 \mathrm{E}+00$ & $3.5 \mathrm{E}+01$ & - & $1.1 \mathrm{E}+00$ \\
NMEA & $10595-95-6$ & $3.5 \mathrm{E}-01$ & $6.4 \mathrm{E}+01$ & $8.9 \mathrm{E}-02$ & $7.1 \mathrm{E}-02$ \\
NPYR & $930-55-2$ & $3.7 \mathrm{E}+00$ & $1.0 \mathrm{E}+03$ & - & $3.7 \mathrm{E}+00$ \\
\hline
\end{tabular}


Table S6. Comparison of boiling point of $N$-Nitrosamines and Haloacetic acids.

\begin{tabular}{lll}
\hline Compounds & Boiling Point $\left({ }^{\circ} \mathbf{C}\right)$ & Data Source \\
\hline$N$-Nitrosamines (NAms) & & \\
$N$-Nitrosodiethylamine (NDEA) & 176 & HSDB \\
$N$-Nitrosodimethylamine (NDMA) & 152 & HSDB \\
$N$-Nitrosodipropylamine (NDPA) & 194.5 & OSHA \\
$N$-Nitrosomethylethylamine (NMEA) & 163 & HSDB \\
$N$-Nitrosopyrrolidine (NPYR) & 214 & HSDB \\
\hline Haloacetic acids (HAAs) & & \\
Chloroacetic acid & 189.3 & HSDB \\
Dichloroacetic acid & 194 & HSDB \\
Trichloroacetic acid & 195.5 & HSDB \\
Bromoacetic acid & 208 & HSDB \\
Dibromoacetic acid & 233 & HSDB \\
\hline
\end{tabular}


Table S7. The Octanol/Water Partition Coefficient of $N$-Nitrosamines, Haloacetic Acids and Trihalomethanes.

\begin{tabular}{clllll}
\hline$N$-Nitrosamines & $N$-nitrosodimethylamine & $N$-nitrosodiethylamine & $N$-nitrosodipropylamine & $N$-nitrosomethylethylamine & $N$-nitrosopyrrolidine \\
$\log \mathbf{K}_{\text {ow }}$ & -0.57 & 0.48 & 1.36 & 0.04 & -0.19 \\
\hline Haloacetic acids & Chloroacetic acid & Dichloroacetic acid & Trichloroacetic acid & Bromoacetic acid & Dibromoacetic acid \\
$\log \mathbf{K}_{\text {ow }}$ & 0.22 & 0.92 & 1.33 & 0.41 & 0.70 \\
\hline Trihalomethanes & Chloroform & Bromodichloromethane & Chlorodibromomethane & Bromoform & - \\
$\log \mathbf{K}_{\text {ow }}$ & 1.97 & 2.00 & 2.16 & 2.40 & - \\
\hline
\end{tabular}

Table S8. Proportion (\%) of different $N$-Nitrosamines in two water treatment plants (WTPs) in total DALYs.

\begin{tabular}{ccccccc}
\hline & WTP-A & & & \multicolumn{3}{c}{ WTP-B } \\
\cline { 6 - 7 } \cline { 5 - 7 } NDMA & NDPA & NMEA & & NDEA & NDMA & NPYR \\
\hline 67.8 & 13.3 & 18.9 & & 77.7 & 15.4 & 6.9
\end{tabular}


Table S9. Concentrations ( $\mu \mathrm{g} / \mathrm{L})$ and patterns of trihalomethanes (THMs) and haloacetic acids (HAAs) in raw water of water treatment plant $A$ and $B$ (WTP-A and WTP-B).

\begin{tabular}{lllll}
\hline DBPs & \multicolumn{3}{c}{ WTP-A } & \multicolumn{2}{c}{ WTP-B } \\
\cline { 2 - 5 } & Mean & SD & Mean & SD \\
\hline THMs & & & & \\
CF & 4.18 & 0.91 & 4.06 & 0.85 \\
BDCM & 0.20 & 0.04 & 0.30 & 0.05 \\
DBCM & ND & ND & ND & ND \\
BF & ND & ND & ND & ND \\
IF & 0.07 & 0.04 & 0.08 & 0.03 \\
HAAs & & & & \\
CAA & 2.10 & 0.17 & 1.60 & 0.08 \\
BCAA & 0.04 & 0.01 & 0.04 & 0.02 \\
BAA & ND & ND & ND & ND \\
DCAA & 0.94 & 0.07 & 0.51 & 0.03 \\
TBAA & ND & ND & ND & ND \\
TCAA & 0.14 & 0.06 & 0.25 & 0.04 \\
BDCAA & 0.15 & 0.03 & 0.37 & 0.03 \\
DBAA & ND & ND & ND & ND \\
CDBAA & 0.33 & 0.04 & 0.28 & 0.06 \\
\hline
\end{tabular}

Abbreviations: $\mathrm{CF}$, chloroform; BDCM, bromodichloromethane; DBCM, dibromomethane; BF, bromoform; IF, iodoform; CAA, chloroacetic acid; BCAA,

bromochloro-acetic acid; BAA, bromoacetic acid; DCAA, dichloroacetic acid; TBAA, tribromoacetic acid; TCAA, trichloroacetic acid; BDCAA, bromodichloroacetic acid; DBAA, dibromoacetic acid; CDBAA, dibromochloroacetic acid; SD, standard deviation; ND, not-detectable. 
Table S10. Estimated lifetime cancer risk (ELCR) of NDMA by age groups and sex under central tendency exposure.

\begin{tabular}{|c|c|c|c|c|c|c|c|c|c|c|c|c|}
\hline \multirow{3}{*}{$\begin{array}{l}\text { Age Group } \\
\text { (year) }\end{array}$} & \multicolumn{4}{|c|}{ ELCR $_{\text {oral }}$} & \multicolumn{4}{|c|}{$\mathbf{E L C R}_{\text {dermal }}$} & \multicolumn{4}{|c|}{ ECLR $_{\text {inhalation }}$} \\
\hline & \multicolumn{2}{|c|}{ WTP-B } & \multicolumn{2}{|c|}{ WTP-A } & \multicolumn{2}{|c|}{ WTP-B } & \multicolumn{2}{|c|}{ WTP-A } & \multicolumn{2}{|c|}{ WTP-B } & \multicolumn{2}{|c|}{ WTP-A } \\
\hline & Male/Female & Total & Male/Female & Total & Male/Female & Total & Male/Female & Total & Male/Female & Total & Male/Female & Total \\
\hline Birth to 0.25 & 7.45E-05/7.33E-05 & $7.36 \mathrm{E}-05$ & $5.26 \mathrm{E}-04 / 5.18 \mathrm{E}-04$ & $5.20 \mathrm{E}-04$ & $1.49 \mathrm{E}-07 / 1.37 \mathrm{E}-07$ & $1.48 \mathrm{E}-07$ & $1.06 \mathrm{E}-06 / 9.68 \mathrm{E}-07$ & $1.05 \mathrm{E}-06$ & $1.11 \mathrm{E}-07 / 8.85 \mathrm{E}-08$ & $1.11 \mathrm{E}-07$ & $7.82 \mathrm{E}-07 / 6.26 \mathrm{E}-07$ & 7.82E-07 \\
\hline 0.25 to 0.5 & $1.16 \mathrm{E}-04 / 1.08 \mathrm{E}-04$ & $1.13 \mathrm{E}-04$ & $8.19 \mathrm{E}-04 / 7.60 \mathrm{E}-04$ & 7.98E-04 & $1.63 \mathrm{E}-07 / 1.72 \mathrm{E}-07$ & $1.67 \mathrm{E}-07$ & $1.16 \mathrm{E}-06 / 1.21 \mathrm{E}-06$ & $1.18 \mathrm{E}-06$ & $1.54 \mathrm{E}-07 / 1.54 \mathrm{E}-07$ & $1.54 \mathrm{E}-07$ & $1.09 \mathrm{E}-06 / 1.09 \mathrm{E}-06$ & $1.09 \mathrm{E}-06$ \\
\hline 0.5 to 0.75 & $1.64 \mathrm{E}-04 / 1.75 \mathrm{E}-04$ & $1.69 \mathrm{E}-04$ & $1.16 \mathrm{E}-03 / 1.23 \mathrm{E}-03$ & $1.19 \mathrm{E}-03$ & $1.62 \mathrm{E}-07 / 1.74 \mathrm{E}-07$ & $1.74 \mathrm{E}-07$ & $1.14 \mathrm{E}-06 / 1.23 \mathrm{E}-06$ & $1.23 \mathrm{E}-06$ & $1.54 \mathrm{E}-07 / 1.77 \mathrm{E}-07$ & $1.77 \mathrm{E}-07$ & $1.09 \mathrm{E}-06 / 1.25 \mathrm{E}-06$ & $1.25 \mathrm{E}-06$ \\
\hline 0.75 to 1 & $2.15 \mathrm{E}-04 / 2.13 \mathrm{E}-04$ & $2.14 \mathrm{E}-04$ & $1.52 \mathrm{E}-03 / 1.50 \mathrm{E}-03$ & $1.52 \mathrm{E}-03$ & 1.68E-07/1.81E-07 & $1.69 \mathrm{E}-07$ & 1.19E-06/1.28E-06 & $1.20 \mathrm{E}-06$ & $1.77 \mathrm{E}-07 / 1.99 \mathrm{E}-07$ & $1.77 \mathrm{E}-07$ & 1.25E-06/1.41E-06 & $1.25 \mathrm{E}-06$ \\
\hline 1 to 2 & $2.10 \mathrm{E}-04 / 2.11 \mathrm{E}-04$ & $2.10 \mathrm{E}-04$ & $1.48 \mathrm{E}-03 / 1.49 \mathrm{E}-03$ & $1.48 \mathrm{E}-03$ & $1.63 \mathrm{E}-07 / 1.67 \mathrm{E}-07$ & $1.64 \mathrm{E}-07$ & $1.14 \mathrm{E}-06 / 1.18 \mathrm{E}-06$ & $1.16 \mathrm{E}-06$ & $1.77 \mathrm{E}-07 / 1.77 \mathrm{E}-07$ & $1.77 \mathrm{E}-07$ & $1.25 \mathrm{E}-06 / 1.25 \mathrm{E}-06$ & $1.25 \mathrm{E}-06$ \\
\hline 2 to 3 & 4.53E-05/4.82E-05 & 4.65E-05 & $3.20 \mathrm{E}-04 / 3.40 \mathrm{E}-04$ & $3.28 \mathrm{E}-04$ & 4.99E-08/5.10E-08 & 5.07E-08 & $3.53 \mathrm{E}-07 / 3.60 \mathrm{E}-07$ & $3.58 \mathrm{E}-07$ & 5.97E-08/5.97E-08 & $5.97 \mathrm{E}-08$ & 4.22E-07/4.22E-07 & 4.22E-07 \\
\hline 3 to 4 & $4.24 \mathrm{E}-05 / 4.39 \mathrm{E}-05$ & $4.29 \mathrm{E}-05$ & $2.99 \mathrm{E}-04 / 3.10 \mathrm{E}-04$ & 3.03E-04 & 4.83E-08/4.91E-08 & $4.89 \mathrm{E}-08$ & $3.41 \mathrm{E}-07 / 3.47 \mathrm{E}-07$ & $3.46 \mathrm{E}-07$ & $5.97 \mathrm{E}-08 / 5.97 \mathrm{E}-08$ & 5.97E-08 & $4.22 \mathrm{E}-07 / 4.22 \mathrm{E}-07$ & 4.22E- 07 \\
\hline 4 to 5 & 3.75E-05/3.73E-05 & 3.73E- 05 & 2.65E-04/2.64E-04 & 2.64E-04 & 4.69E-08/4.77E-08 & 4.68E-08 & 3.32E-07/3.37E-07 & 3.31E-07 & 5.97E-08/5.97E-08 & 5.97E-08 & 4.22E-07/4.22E-07 & $4.22 \mathrm{E}-07$ \\
\hline 5 to 6 & $3.39 \mathrm{E}-05 / 3.42 \mathrm{E}-05$ & $3.40 \mathrm{E}-05$ & $2.40 \mathrm{E}-04 / 2.42 \mathrm{E}-04$ & $2.41 \mathrm{E}-04$ & $4.30 \mathrm{E}-08 / 4.61 \mathrm{E}-08$ & $4.57 \mathrm{E}-08$ & $3.04 \mathrm{E}-07 / 3.26 \mathrm{E}-07$ & 3.24E-07 & $5.30 \mathrm{E}-08 / 5.97 \mathrm{E}-08$ & 5.97E-08 & $3.75 \mathrm{E}-07 / 4.22 \mathrm{E}-07$ & $4.22 \mathrm{E}-07$ \\
\hline 6 to 9 & $3.42 \mathrm{E}-05 / 3.54 \mathrm{E}-05$ & $3.47 \mathrm{E}-05$ & 2.42E-04/2.50E-04 & $2.45 \mathrm{E}-04$ & 4.15E-08/4.49E-08 & $4.19 \mathrm{E}-08$ & $2.93 \mathrm{E}-07 / 3.17 \mathrm{E}-07$ & $2.96 \mathrm{E}-07$ & $5.97 \mathrm{E}-08 / 6.63 \mathrm{E}-08$ & 5.97E-08 & 4.22E-07/4.69E-07 & $4.22 \mathrm{E}-07$ \\
\hline 9 to 12 & 2.66E-05/2.75E-05 & $2.70 \mathrm{E}-05$ & $1.89 \mathrm{E}-04 / 1.94 \mathrm{E}-04$ & $1.91 \mathrm{E}-04$ & 3.91E-08/4.23E-08 & 3.95E-08 & 2.76E-07/2.99E-07 & $2.79 \mathrm{E}-07$ & $6.63 \mathrm{E}-08 / 7.30 \mathrm{E}-08$ & $6.63 \mathrm{E}-08$ & 4.69E-07/5.16E-07 & 4.69E-07 \\
\hline 12 to 15 & $2.25 \mathrm{E}-05 / 2.30 \mathrm{E}-05$ & $2.27 \mathrm{E}-05$ & $1.58 \mathrm{E}-04 / 1.62 \mathrm{E}-04$ & $1.60 \mathrm{E}-04$ & 3.77E-08/4.22E-08 & 3.99E-08 & $2.66 \mathrm{E}-07 / 2.98 \mathrm{E}-07$ & $2.82 \mathrm{E}-07$ & $7.30 \mathrm{E}-08 / 8.63 \mathrm{E}-08$ & 7.97E-08 & $5.16 \mathrm{E}-07 / 6.09 \mathrm{E}-07$ & $5.62 \mathrm{E}-07$ \\
\hline 15 to 18 & $2.04 \mathrm{E}-05 / 1.95 \mathrm{E}-05$ & $2.00 \mathrm{E}-05$ & $1.44 \mathrm{E}-04 / 1.38 \mathrm{E}-04$ & $1.42 \mathrm{E}-04$ & $3.25 \mathrm{E}-08 / 3.88 \mathrm{E}-08$ & 3.47E-08 & $2.30 \mathrm{E}-07 / 2.75 \mathrm{E}-07$ & $2.45 \mathrm{E}-07$ & $5.97 \mathrm{E}-08 / 7.97 \mathrm{E}-08$ & 6.63E-08 & $4.22 \mathrm{E}-07 / 5.62 \mathrm{E}-07$ & $4.69 \mathrm{E}-07$ \\
\hline 18 to 45 & 7.91E-06/8.14E-06 & $8.07 \mathrm{E}-06$ & $5.59 \mathrm{E}-05 / 5.76 \mathrm{E}-05$ & 5.70E-05 & $9.17 \mathrm{E}-09 / 9.50 \mathrm{E}-09$ & $9.38 \mathrm{E}-09$ & $6.49 \mathrm{E}-08 / 6.71 \mathrm{E}-08$ & $6.63 \mathrm{E}-08$ & $1.77 \mathrm{E}-08 / 1.77 \mathrm{E}-08$ & $1.77 \mathrm{E}-08$ & $1.25 \mathrm{E}-07 / 1.25 \mathrm{E}-07$ & $1.25 \mathrm{E}-07$ \\
\hline 45 to 60 & 7.94E-06/7.61E-06 & $7.87 \mathrm{E}-06$ & $5.60 \mathrm{E}-05 / 5.37 \mathrm{E}-05$ & $5.56 \mathrm{E}-05$ & $8.48 \mathrm{E}-09 / 8.86 \mathrm{E}-09$ & $8.45 \mathrm{E}-09$ & $6.00 \mathrm{E}-08 / 6.27 \mathrm{E}-08$ & $5.97 \mathrm{E}-08$ & $1.54 \mathrm{E}-08 / 1.54 \mathrm{E}-08$ & $1.54 \mathrm{E}-08$ & $1.09 \mathrm{E}-07 / 1.09 \mathrm{E}-07$ & $1.09 \mathrm{E}-07$ \\
\hline 60 to 80 & $8.08 \mathrm{E}-06 / 7.54 \mathrm{E}-06$ & $7.83 \mathrm{E}-06$ & $5.71 \mathrm{E}-05 / 5.33 \mathrm{E}-05$ & $5.54 \mathrm{E}-05$ & 7.82E-09/9.34E-09 & $8.22 \mathrm{E}-09$ & $5.53 \mathrm{E}-08 / 6.59 \mathrm{E}-08$ & 5.81E-08 & $1.33 \mathrm{E}-08 / 1.33 \mathrm{E}-08$ & $1.33 \mathrm{E}-08$ & $9.37 \mathrm{E}-08 / 9.37 \mathrm{E}-08$ & $9.37 \mathrm{E}-08$ \\
\hline$\geq 80$ & 7.87E-06/6.97E-06 & $7.26 \mathrm{E}-06$ & $5.57 \mathrm{E}-05 / 4.93 \mathrm{E}-05$ & $5.13 \mathrm{E}-05$ & 7.54E-09/7.65E-09 & 7.69E-09 & $5.33 \mathrm{E}-08 / 5.41 \mathrm{E}-08$ & 5.44E-08 & $1.11 \mathrm{E}-08 / 1.11 \mathrm{E}-08$ & $1.11 \mathrm{E}-08$ & $7.82 \mathrm{E}-08 / 7.82 \mathrm{E}-08$ & $7.82 \mathrm{E}-08$ \\
\hline
\end{tabular}


Table S11. Estimated lifetime cancer risk (ELCR) of NDMA by age groups and sex under reasonable maximum exposure.

\begin{tabular}{|c|c|c|c|c|c|c|c|c|c|c|c|c|}
\hline \multirow{3}{*}{$\begin{array}{l}\text { Age Group } \\
\text { (year) }\end{array}$} & \multicolumn{4}{|c|}{ ELCR $_{\text {oral }}$} & \multicolumn{4}{|c|}{$\mathbf{E L C R}_{\text {dermal }}$} & \multicolumn{4}{|c|}{$\mathbf{E C L R}_{\text {inhalation }}$} \\
\hline & \multicolumn{2}{|c|}{ WTP-B } & \multicolumn{2}{|c|}{ WTP-A } & \multicolumn{2}{|c|}{ WTP-B } & \multicolumn{2}{|c|}{ WTP-A } & \multicolumn{2}{|c|}{ WTP-B } & \multicolumn{2}{|c|}{ WTP-A } \\
\hline & Male/Female & Total & Male/Female & Total & Male/Female & Total & Male/Female & Total & Male/Female & Total & Male/Female & Total \\
\hline Birth to 0.25 & 3.04E-04/3.79E-04 & $3.33 \mathrm{E}-04$ & $1.88 \mathrm{E}-03 / 2.35 \mathrm{E}-03$ & $2.05 \mathrm{E}-03$ & 4.39E-07/4.63E-07 & $4.59 \mathrm{E}-07$ & $2.71 \mathrm{E}-06 / 2.86 \mathrm{E}-06$ & $2.84 \mathrm{E}-06$ & 4.85E-07/4.55E-07 & $4.85 \mathrm{E}-07$ & $3.00 \mathrm{E}-06 / 2.81 \mathrm{E}-06$ & $3.00 \mathrm{E}-06$ \\
\hline 0.25 to 0.5 & $5.34 \mathrm{E}-04 / 4.98 \mathrm{E}-04$ & $5.39 \mathrm{E}-04$ & $3.29 \mathrm{E}-03 / 3.08 \mathrm{E}-03$ & 3.33E- 03 & 4.03E-07/4.32E-07 & $4.15 \mathrm{E}-07$ & $2.49 \mathrm{E}-06 / 2.66 \mathrm{E}-06$ & $2.56 \mathrm{E}-06$ & $4.85 \mathrm{E}-07 / 5.15 \mathrm{E}-07$ & $4.85 \mathrm{E}-07$ & $3.00 \mathrm{E}-06 / 3.18 \mathrm{E}-06$ & $3.00 \mathrm{E}-06$ \\
\hline 0.5 to 0.75 & $6.22 \mathrm{E}-04 / 6.62 \mathrm{E}-04$ & $6.33 \mathrm{E}-04$ & $3.84 \mathrm{E}-03 / 4.09 \mathrm{E}-03$ & $3.91 \mathrm{E}-03$ & 4.16E-07/4.27E-07 & $4.25 \mathrm{E}-07$ & $2.57 \mathrm{E}-06 / 2.64 \mathrm{E}-06$ & 2.63E- 06 & $5.46 \mathrm{E}-07 / 5.77 \mathrm{E}-07$ & $5.77 \mathrm{E}-07$ & 3.37E-06/3.56E-06 & $3.56 \mathrm{E}-06$ \\
\hline 0.75 to 1 & $8.86 \mathrm{E}-04 / 6.53 \mathrm{E}-04$ & $8.13 \mathrm{E}-04$ & $5.47 \mathrm{E}-03 / 4.03 \mathrm{E}-03$ & $5.02 \mathrm{E}-03$ & 4.16E-07/4.26E-07 & $4.20 \mathrm{E}-07$ & $2.57 \mathrm{E}-06 / 2.63 \mathrm{E}-06$ & $2.59 \mathrm{E}-06$ & $6.06 \mathrm{E}-07 / 6.06 \mathrm{E}-07$ & $6.06 \mathrm{E}-07$ & $3.75 \mathrm{E}-06 / 3.75 \mathrm{E}-06$ & $3.75 \mathrm{E}-06$ \\
\hline 1 to 2 & $6.94 \mathrm{E}-04 / 6.56 \mathrm{E}-04$ & $6.72 \mathrm{E}-04$ & 4.28E-03/4.06E-03 & $4.15 \mathrm{E}-03$ & 4.01E-07/4.14E-07 & $4.06 \mathrm{E}-07$ & $2.47 \mathrm{E}-06 / 2.55 \mathrm{E}-06$ & $2.50 \mathrm{E}-06$ & $5.77 \mathrm{E}-07 / 5.77 \mathrm{E}-07$ & $5.77 \mathrm{E}-07$ & 3.56E-06/3.56E-06 & $3.56 \mathrm{E}-06$ \\
\hline 2 to 3 & $1.28 \mathrm{E}-04 / 1.40 \mathrm{E}-04$ & $1.32 \mathrm{E}-04$ & 7.87E-04/8.68E-04 & $8.15 \mathrm{E}-04$ & $1.16 \mathrm{E}-07 / 1.18 \mathrm{E}-07$ & $1.17 \mathrm{E}-07$ & 7.14E-07/7.31E-07 & 7.25E-07 & $1.73 \mathrm{E}-07 / 1.73 \mathrm{E}-07$ & $1.73 \mathrm{E}-07$ & $1.07 \mathrm{E}-06 / 1.07 \mathrm{E}-06$ & $1.07 \mathrm{E}-06$ \\
\hline 3 to 4 & $1.17 \mathrm{E}-04 / 1.22 \mathrm{E}-04$ & $1.20 \mathrm{E}-04$ & 7.27E-04/7.51E-04 & $7.38 \mathrm{E}-04$ & $1.10 \mathrm{E}-07 / 1.15 \mathrm{E}-07$ & $1.13 \mathrm{E}-07$ & $6.80 \mathrm{E}-07 / 7.11 \mathrm{E}-07$ & $6.98 \mathrm{E}-07$ & $1.64 \mathrm{E}-07 / 1.82 \mathrm{E}-07$ & $1.73 \mathrm{E}-07$ & $1.01 \mathrm{E}-06 / 1.13 \mathrm{E}-06$ & $1.07 \mathrm{E}-06$ \\
\hline 4 to 5 & $1.04 \mathrm{E}-04 / 1.07 \mathrm{E}-04$ & $1.05 \mathrm{E}-04$ & 6.42E-04/6.60E-04 & $6.49 \mathrm{E}-04$ & $1.14 \mathrm{E}-07 / 1.12 \mathrm{E}-07$ & $1.11 \mathrm{E}-07$ & 7.03E-07/6.89E-07 & $6.86 \mathrm{E}-07$ & $1.82 \mathrm{E}-07 / 1.73 \mathrm{E}-07$ & $1.73 \mathrm{E}-07$ & $1.13 \mathrm{E}-06 / 1.07 \mathrm{E}-06$ & $1.07 \mathrm{E}-06$ \\
\hline 5 to 6 & $9.35 \mathrm{E}-05 / 9.70 \mathrm{E}-05$ & $9.49 \mathrm{E}-05$ & $5.77 \mathrm{E}-04 / 5.98 \mathrm{E}-04$ & $5.86 \mathrm{E}-04$ & $1.04 \mathrm{E}-07 / 1.09 \mathrm{E}-07$ & $1.05 \mathrm{E}-07$ & $6.40 \mathrm{E}-07 / 6.74 \mathrm{E}-07$ & $6.50 \mathrm{E}-07$ & $1.64 \mathrm{E}-07 / 1.73 \mathrm{E}-07$ & $1.64 \mathrm{E}-07$ & $1.01 \mathrm{E}-06 / 1.07 \mathrm{E}-06$ & $1.01 \mathrm{E}-06$ \\
\hline 6 to 9 & 8.37E-05/8.97E-05 & $8.62 \mathrm{E}-05$ & $5.17 \mathrm{E}-04 / 5.53 \mathrm{E}-04$ & 5.33E-04 & $1.07 \mathrm{E}-07 / 1.08 \mathrm{E}-07$ & $1.08 \mathrm{E}-07$ & $6.58 \mathrm{E}-07 / 6.65 \mathrm{E}-07$ & 6.67E- 07 & $1.82 \mathrm{E}-07 / 1.82 \mathrm{E}-07$ & $1.82 \mathrm{E}-07$ & $1.13 \mathrm{E}-06 / 1.13 \mathrm{E}-06$ & $1.13 \mathrm{E}-06$ \\
\hline 9 to 12 & $6.47 \mathrm{E}-05 / 6.81 \mathrm{E}-05$ & $6.64 \mathrm{E}-05$ & $4.00 \mathrm{E}-04 / 4.21 \mathrm{E}-04$ & 4.10E-04 & $1.00 \mathrm{E}-07 / 1.06 \mathrm{E}-07$ & $1.03 \mathrm{E}-07$ & $6.17 \mathrm{E}-07 / 6.54 \mathrm{E}-07$ & $6.37 \mathrm{E}-07$ & $1.91 \mathrm{E}-07 / 2.10 \mathrm{E}-07$ & $2.01 \mathrm{E}-07$ & $1.18 \mathrm{E}-06 / 1.29 \mathrm{E}-06$ & $1.24 \mathrm{E}-06$ \\
\hline 12 to 15 & $5.89 \mathrm{E}-05 / 6.26 \mathrm{E}-05$ & $6.07 \mathrm{E}-05$ & $3.64 \mathrm{E}-04 / 3.86 \mathrm{E}-04$ & 3.75E-04 & $9.58 \mathrm{E}-08 / 1.04 \mathrm{E}-07$ & $1.03 \mathrm{E}-07$ & $5.92 \mathrm{E}-07 / 6.44 \mathrm{E}-07$ & $6.33 \mathrm{E}-07$ & $2.19 \mathrm{E}-07 / 2.73 \mathrm{E}-07$ & $2.55 \mathrm{E}-07$ & $1.35 \mathrm{E}-06 / 1.68 \mathrm{E}-06$ & $1.57 \mathrm{E}-06$ \\
\hline 15 to 18 & $6.24 \mathrm{E}-05 / 6.40 \mathrm{E}-05$ & $6.31 \mathrm{E}-05$ & $3.85 \mathrm{E}-04 / 3.95 \mathrm{E}-04$ & 3.89E-04 & 8.34E-08/9.47E-08 & $9.06 \mathrm{E}-08$ & $5.15 \mathrm{E}-07 / 5.84 \mathrm{E}-07$ & $5.59 \mathrm{E}-07$ & $2.10 \mathrm{E}-07 / 2.64 \mathrm{E}-07$ & $2.37 \mathrm{E}-07$ & $1.29 \mathrm{E}-06 / 1.63 \mathrm{E}-06$ & $1.46 \mathrm{E}-06$ \\
\hline 18 to 45 & $2.94 \mathrm{E}-05 / 3.12 \mathrm{E}-05$ & $3.10 \mathrm{E}-05$ & $1.82 \mathrm{E}-04 / 1.92 \mathrm{E}-04$ & $1.92 \mathrm{E}-04$ & $2.22 \mathrm{E}-08 / 2.47 \mathrm{E}-08$ & $2.36 \mathrm{E}-08$ & $1.37 \mathrm{E}-07 / 1.53 \mathrm{E}-07$ & $1.45 \mathrm{E}-07$ & $5.46 \mathrm{E}-08 / 6.06 \mathrm{E}-08$ & $5.77 \mathrm{E}-08$ & 3.37E-07/3.75E-07 & $3.56 \mathrm{E}-07$ \\
\hline 45 to 60 & $3.06 \mathrm{E}-05 / 2.89 \mathrm{E}-05$ & $3.04 \mathrm{E}-05$ & $1.89 \mathrm{E}-04 / 1.79 \mathrm{E}-04$ & $1.88 \mathrm{E}-04$ & $2.13 \mathrm{E}-08 / 2.19 \mathrm{E}-08$ & $2.21 \mathrm{E}-08$ & $1.31 \mathrm{E}-07 / 1.35 \mathrm{E}-07$ & $1.36 \mathrm{E}-07$ & $5.15 \mathrm{E}-08 / 5.46 \mathrm{E}-08$ & 5.46E-08 & $3.18 \mathrm{E}-07 / 3.37 \mathrm{E}-07$ & $3.37 \mathrm{E}-07$ \\
\hline 60 to 80 & $2.99 \mathrm{E}-05 / 2.76 \mathrm{E}-05$ & $2.89 \mathrm{E}-05$ & $1.84 \mathrm{E}-04 / 1.70 \mathrm{E}-04$ & $1.79 \mathrm{E}-04$ & $2.02 \mathrm{E}-08 / 2.18 \mathrm{E}-08$ & $2.12 \mathrm{E}-08$ & $1.25 \mathrm{E}-07 / 1.34 \mathrm{E}-07$ & $1.31 \mathrm{E}-07$ & 4.55E-08/4.85E-08 & 4.55E- 08 & $2.81 \mathrm{E}-07 / 3.00 \mathrm{E}-07$ & $2.81 \mathrm{E}-07$ \\
\hline$\geq 80$ & 3.00E-05/2.73E-05 & $2.94 \mathrm{E}-05$ & $1.85 \mathrm{E}-04 / 1.69 \mathrm{E}-04$ & $1.81 \mathrm{E}-04$ & 2.32E-08/2.20E-08 & $2.27 \mathrm{E}-08$ & $1.43 \mathrm{E}-07 / 1.36 \mathrm{E}-07$ & $1.40 \mathrm{E}-07$ & $5.46 \mathrm{E}-08 / 4.55 \mathrm{E}-08$ & $4.85 \mathrm{E}-08$ & $3.37 \mathrm{E}-07 / 2.81 \mathrm{E}-07$ & $3.00 \mathrm{E}-07$ \\
\hline
\end{tabular}


Table S12. Estimated lifetime cancer risk (ELCR) of NDEA by age groups and sex under central tendency exposure.

\begin{tabular}{|c|c|c|c|c|c|c|c|c|c|}
\hline \multirow{2}{*}{$\begin{array}{l}\text { Age Group } \\
\text { (yeas) }\end{array}$} & \multicolumn{3}{|c|}{ ELCR oral } & \multicolumn{3}{|c|}{$\mathbf{E L C R}_{\text {dermal }}$} & \multicolumn{3}{|c|}{$\mathbf{E C L R}_{\text {inhalation }}$} \\
\hline & Male & Female & Total & Male & Female & Total & Male & Female & Total \\
\hline Birth to 0.25 & $3.13 \mathrm{E}-04$ & $3.08 \mathrm{E}-04$ & $3.10 \mathrm{E}-04$ & $3.06 \mathrm{E}-06$ & $2.81 \mathrm{E}-06$ & 3.03E-06 & 2.79E-06 & $2.23 \mathrm{E}-06$ & $2.79 \mathrm{E}-06$ \\
\hline 0.25 to 0.5 & $4.88 \mathrm{E}-04$ & 4.53E-04 & 4.76E-04 & 3.34E-06 & $3.51 \mathrm{E}-06$ & $3.42 \mathrm{E}-06$ & 3.90E-06 & $3.90 \mathrm{E}-06$ & $3.90 \mathrm{E}-06$ \\
\hline 0.5 to 0.75 & $6.90 \mathrm{E}-04$ & 7.36E-04 & 7.09E-04 & $3.31 \mathrm{E}-06$ & 3.57E-06 & $3.57 \mathrm{E}-06$ & 3.90E-06 & 4.46E-06 & 4.46E-06 \\
\hline 0.75 to 1 & $9.06 \mathrm{E}-04$ & 8.98E-04 & $9.06 \mathrm{E}-04$ & 3.44E-06 & $3.71 \mathrm{E}-06$ & $3.46 \mathrm{E}-06$ & 4.46E-06 & $5.02 \mathrm{E}-06$ & 4.46E-06 \\
\hline 1 to 2 & 8.89E-04 & 8.89E-04 & 8.89E-04 & 3.33E-06 & $3.41 \mathrm{E}-06$ & 3.36E-06 & 4.46E-06 & 4.46E-06 & 4.46E-06 \\
\hline 2 to 3 & $1.91 \mathrm{E}-04$ & $2.03 \mathrm{E}-04$ & $1.96 \mathrm{E}-04$ & $1.03 \mathrm{E}-06$ & $1.04 \mathrm{E}-06$ & $1.04 \mathrm{E}-06$ & $1.50 \mathrm{E}-06$ & $1.50 \mathrm{E}-06$ & $1.50 \mathrm{E}-06$ \\
\hline 3 to 4 & $1.78 \mathrm{E}-04$ & $1.85 \mathrm{E}-04$ & $1.80 \mathrm{E}-04$ & $9.93 \mathrm{E}-07$ & $1.01 \mathrm{E}-06$ & $1.00 \mathrm{E}-06$ & $1.50 \mathrm{E}-06$ & $1.50 \mathrm{E}-06$ & $1.50 \mathrm{E}-06$ \\
\hline 4 to 5 & $1.58 \mathrm{E}-04$ & $1.57 \mathrm{E}-04$ & $1.57 \mathrm{E}-04$ & $9.67 \mathrm{E}-07$ & $9.75 \mathrm{E}-07$ & $9.58 \mathrm{E}-07$ & $1.50 \mathrm{E}-06$ & $1.50 \mathrm{E}-06$ & $1.50 \mathrm{E}-06$ \\
\hline 5 to 6 & $1.42 \mathrm{E}-04$ & $1.44 \mathrm{E}-04$ & $1.43 \mathrm{E}-04$ & 8.81E-07 & $9.50 \mathrm{E}-07$ & $9.41 \mathrm{E}-07$ & $1.34 \mathrm{E}-06$ & $1.50 \mathrm{E}-06$ & $1.50 \mathrm{E}-06$ \\
\hline 6 to 9 & $1.44 \mathrm{E}-04$ & $1.48 \mathrm{E}-04$ & $1.46 \mathrm{E}-04$ & $8.50 \mathrm{E}-07$ & $9.24 \mathrm{E}-07$ & $8.59 \mathrm{E}-07$ & $1.50 \mathrm{E}-06$ & $1.67 \mathrm{E}-06$ & $1.50 \mathrm{E}-06$ \\
\hline 9 to 12 & $1.12 \mathrm{E}-04$ & $1.16 \mathrm{E}-04$ & $1.14 \mathrm{E}-04$ & 8.01E-07 & $8.63 \mathrm{E}-07$ & $8.10 \mathrm{E}-07$ & $1.67 \mathrm{E}-06$ & $1.84 \mathrm{E}-06$ & $1.67 \mathrm{E}-06$ \\
\hline 12 to 15 & $9.41 \mathrm{E}-05$ & $9.67 \mathrm{E}-05$ & $9.58 \mathrm{E}-05$ & 7.73E-07 & 8.63E-07 & 8.19E-07 & $1.84 \mathrm{E}-06$ & $2.18 \mathrm{E}-06$ & $2.01 \mathrm{E}-06$ \\
\hline 15 to 18 & $8.58 \mathrm{E}-05$ & $8.22 \mathrm{E}-05$ & $8.43 \mathrm{E}-05$ & $6.66 \mathrm{E}-07$ & 7.97E-07 & $7.12 \mathrm{E}-07$ & $1.50 \mathrm{E}-06$ & $2.01 \mathrm{E}-06$ & $1.67 \mathrm{E}-06$ \\
\hline 18 to 45 & 3.33E-05 & $3.43 \mathrm{E}-05$ & $3.40 \mathrm{E}-05$ & $1.88 \mathrm{E}-07$ & $1.95 \mathrm{E}-07$ & $1.93 \mathrm{E}-07$ & $4.46 \mathrm{E}-07$ & 4.46E-07 & $4.46 \mathrm{E}-07$ \\
\hline 45 to 60 & $3.34 \mathrm{E}-05$ & $3.20 \mathrm{E}-05$ & $3.31 \mathrm{E}-05$ & $1.74 \mathrm{E}-07$ & $1.81 \mathrm{E}-07$ & $1.74 \mathrm{E}-07$ & $3.90 \mathrm{E}-07$ & $3.90 \mathrm{E}-07$ & $3.90 \mathrm{E}-07$ \\
\hline 60 to 80 & $3.40 \mathrm{E}-05$ & $3.18 \mathrm{E}-05$ & $3.30 \mathrm{E}-05$ & $1.61 \mathrm{E}-07$ & $1.92 \mathrm{E}-07$ & $1.68 \mathrm{E}-07$ & $3.35 \mathrm{E}-07$ & $3.35 \mathrm{E}-07$ & $3.35 \mathrm{E}-07$ \\
\hline$\geq 80$ & $3.31 \mathrm{E}-05$ & $2.94 \mathrm{E}-05$ & $3.06 \mathrm{E}-05$ & $1.55 \mathrm{E}-07$ & $1.57 \mathrm{E}-07$ & $1.58 \mathrm{E}-07$ & $2.79 \mathrm{E}-07$ & 2.79E-07 & $2.79 \mathrm{E}-07$ \\
\hline
\end{tabular}


Table S13. Estimated lifetime cancer risk (ELCR) of NDEA by age groups and sex under reasonable maximum exposure.

\begin{tabular}{|c|c|c|c|c|c|c|c|c|c|}
\hline \multirow{2}{*}{$\begin{array}{l}\text { Age Group } \\
\text { (yeas) }\end{array}$} & \multicolumn{3}{|c|}{ ELCR oral } & \multicolumn{3}{|c|}{$\mathbf{E L C R}_{\text {dermal }}$} & \multicolumn{3}{|c|}{$\mathbf{E C L R}_{\text {inhalation }}$} \\
\hline & Male & Female & Total & Male & Female & Total & Male & Female & Total \\
\hline Birth to 0.25 & $1.10 \mathrm{E}-03$ & $1.37 \mathrm{E}-03$ & $1.20 \mathrm{E}-03$ & 7.74E-06 & $8.18 \mathrm{E}-06$ & $8.10 \mathrm{E}-06$ & $1.05 \mathrm{E}-05$ & $9.87 \mathrm{E}-06$ & $1.05 \mathrm{E}-05$ \\
\hline 0.25 to 0.5 & $1.94 \mathrm{E}-03$ & $1.81 \mathrm{E}-03$ & $1.96 \mathrm{E}-03$ & 7.11E-06 & 7.63E-06 & 7.33E-06 & $1.05 \mathrm{E}-05$ & $1.12 \mathrm{E}-05$ & $1.05 \mathrm{E}-05$ \\
\hline 0.5 to 0.75 & $2.26 \mathrm{E}-03$ & $2.40 \mathrm{E}-03$ & $2.30 \mathrm{E}-03$ & $7.35 \mathrm{E}-06$ & 7.56E-06 & $7.51 \mathrm{E}-06$ & $1.18 \mathrm{E}-05$ & $1.25 \mathrm{E}-05$ & $1.25 \mathrm{E}-05$ \\
\hline 0.75 to 1 & $3.21 \mathrm{E}-03$ & $2.37 \mathrm{E}-03$ & $2.95 \mathrm{E}-03$ & 7.35E-06 & 7.53E-06 & 7.43E-06 & $1.31 \mathrm{E}-05$ & $1.31 \mathrm{E}-05$ & $1.31 \mathrm{E}-05$ \\
\hline 1 to 2 & $2.52 \mathrm{E}-03$ & $2.38 \mathrm{E}-03$ & $2.44 \mathrm{E}-03$ & 7.08E-06 & 7.31E-06 & 7.16E-06 & $1.25 \mathrm{E}-05$ & $1.25 \mathrm{E}-05$ & $1.25 \mathrm{E}-05$ \\
\hline 2 to 3 & 4.63E-04 & $5.09 \mathrm{E}-04$ & 4.79E-04 & $2.05 \mathrm{E}-06$ & $2.09 \mathrm{E}-06$ & $2.08 \mathrm{E}-06$ & $3.75 \mathrm{E}-06$ & $3.75 \mathrm{E}-06$ & $3.75 \mathrm{E}-06$ \\
\hline 3 to 4 & 4.27E-04 & 4.42E-04 & 4.33E-04 & $1.95 \mathrm{E}-06$ & $2.04 \mathrm{E}-06$ & $2.00 \mathrm{E}-06$ & $3.55 \mathrm{E}-06$ & $3.95 \mathrm{E}-06$ & $3.75 \mathrm{E}-06$ \\
\hline 4 to 5 & $3.77 \mathrm{E}-04$ & $3.88 \mathrm{E}-04$ & $3.81 \mathrm{E}-04$ & $2.01 \mathrm{E}-06$ & $1.97 \mathrm{E}-06$ & $1.96 \mathrm{E}-06$ & $3.95 \mathrm{E}-06$ & $3.75 \mathrm{E}-06$ & $3.75 \mathrm{E}-06$ \\
\hline 5 to 6 & 3.39E-04 & $3.51 \mathrm{E}-04$ & $3.44 \mathrm{E}-04$ & $1.84 \mathrm{E}-06$ & $1.93 \mathrm{E}-06$ & $1.86 \mathrm{E}-06$ & $3.55 \mathrm{E}-06$ & $3.75 \mathrm{E}-06$ & $3.55 \mathrm{E}-06$ \\
\hline 6 to 9 & 3.04E-04 & $3.25 \mathrm{E}-04$ & $3.13 \mathrm{E}-04$ & $1.89 \mathrm{E}-06$ & $1.91 \mathrm{E}-06$ & $1.91 \mathrm{E}-06$ & $3.95 \mathrm{E}-06$ & $3.95 \mathrm{E}-06$ & $3.95 \mathrm{E}-06$ \\
\hline 9 to 12 & $2.35 \mathrm{E}-04$ & $2.47 \mathrm{E}-04$ & $2.41 \mathrm{E}-04$ & $1.77 \mathrm{E}-06$ & $1.87 \mathrm{E}-06$ & $1.83 \mathrm{E}-06$ & 4.14E-06 & $4.55 \mathrm{E}-06$ & $4.35 \mathrm{E}-06$ \\
\hline 12 to 15 & 2.14E-04 & $2.27 \mathrm{E}-04$ & $2.20 \mathrm{E}-04$ & $1.70 \mathrm{E}-06$ & $1.85 \mathrm{E}-06$ & $1.81 \mathrm{E}-06$ & 4.74E-06 & $5.92 \mathrm{E}-06$ & $5.53 \mathrm{E}-06$ \\
\hline 15 to 18 & $2.27 \mathrm{E}-04$ & $2.32 \mathrm{E}-04$ & 2.29E-04 & $1.48 \mathrm{E}-06$ & $1.68 \mathrm{E}-06$ & $1.60 \mathrm{E}-06$ & 4.55E-06 & $5.73 \mathrm{E}-06$ & $5.14 \mathrm{E}-06$ \\
\hline 18 to 45 & $1.06 \mathrm{E}-04$ & $1.13 \mathrm{E}-04$ & $1.12 \mathrm{E}-04$ & $3.91 \mathrm{E}-07$ & 4.37E-07 & $4.15 \mathrm{E}-07$ & $1.18 \mathrm{E}-06$ & $1.31 \mathrm{E}-06$ & $1.25 \mathrm{E}-06$ \\
\hline 45 to 60 & $1.11 \mathrm{E}-04$ & $1.04 \mathrm{E}-04$ & $1.10 \mathrm{E}-04$ & $3.76 \mathrm{E}-07$ & $3.87 \mathrm{E}-07$ & $3.89 \mathrm{E}-07$ & $1.12 \mathrm{E}-06$ & $1.18 \mathrm{E}-06$ & $1.18 \mathrm{E}-06$ \\
\hline 60 to 80 & $1.08 \mathrm{E}-04$ & $9.99 \mathrm{E}-05$ & $1.05 \mathrm{E}-04$ & $3.55 \mathrm{E}-07$ & $3.83 \mathrm{E}-07$ & $3.73 \mathrm{E}-07$ & $9.87 \mathrm{E}-07$ & $1.05 \mathrm{E}-06$ & $9.87 \mathrm{E}-07$ \\
\hline$\geq 80$ & $1.08 \mathrm{E}-04$ & 9.93E-05 & $1.06 \mathrm{E}-04$ & 4.11E-07 & 3.89E-07 & 3.99E-07 & $1.18 \mathrm{E}-06$ & $9.87 \mathrm{E}-07$ & $1.05 \mathrm{E}-06$ \\
\hline
\end{tabular}


Table S14. Estimated lifetime cancer risk (ELCR) of NDPA by age groups and sex under central tendency exposure.

\begin{tabular}{|c|c|c|c|c|c|c|c|c|c|}
\hline \multirow{2}{*}{$\begin{array}{l}\text { Age Group } \\
\text { (yeas) }\end{array}$} & \multicolumn{3}{|c|}{ ELCR $_{\text {oral }}$} & \multicolumn{3}{|c|}{ ELCR $_{\text {dermal }}$} & \multicolumn{3}{|c|}{ ECLR $_{\text {inhalation }}$} \\
\hline & Male & Female & Total & Male & Female & Total & Male & Female & Total \\
\hline Birth to 0.25 & 8.59E-05 & $8.45 \mathrm{E}-05$ & $8.48 \mathrm{E}-05$ & $2.63 \mathrm{E}-06$ & $2.41 \mathrm{E}-06$ & $2.60 \mathrm{E}-06$ & $6.25 \mathrm{E}-07$ & $5.00 \mathrm{E}-07$ & $6.25 \mathrm{E}-07$ \\
\hline 0.25 to 0.5 & $1.33 \mathrm{E}-04$ & $1.24 \mathrm{E}-04$ & $1.30 \mathrm{E}-04$ & $2.86 \mathrm{E}-06$ & $3.01 \mathrm{E}-06$ & $2.94 \mathrm{E}-06$ & $8.75 \mathrm{E}-07$ & $8.75 \mathrm{E}-07$ & $8.75 \mathrm{E}-07$ \\
\hline 0.5 to 0.75 & $1.89 \mathrm{E}-04$ & $2.01 \mathrm{E}-04$ & $1.94 \mathrm{E}-04$ & $2.83 \mathrm{E}-06$ & 3.07E-06 & 3.07E-06 & $8.75 \mathrm{E}-07$ & $9.97 \mathrm{E}-07$ & $9.97 \mathrm{E}-07$ \\
\hline 0.75 to 1 & $2.48 \mathrm{E}-04$ & $2.46 \mathrm{E}-04$ & $2.48 \mathrm{E}-04$ & $2.95 \mathrm{E}-06$ & $3.18 \mathrm{E}-06$ & $2.97 \mathrm{E}-06$ & $9.97 \mathrm{E}-07$ & $1.13 \mathrm{E}-06$ & 9.97E-07 \\
\hline 1 to 2 & $2.43 \mathrm{E}-04$ & $2.43 \mathrm{E}-04$ & $2.42 \mathrm{E}-04$ & $2.85 \mathrm{E}-06$ & 2.93E-06 & $2.88 \mathrm{E}-06$ & 9.97E-07 & $9.97 \mathrm{E}-07$ & $9.97 \mathrm{E}-07$ \\
\hline 2 to 3 & $5.23 \mathrm{E}-05$ & $5.55 \mathrm{E}-05$ & $5.36 \mathrm{E}-05$ & $8.78 \mathrm{E}-07$ & 8.96E-07 & 8.91E-07 & $3.37 \mathrm{E}-07$ & $3.37 \mathrm{E}-07$ & $3.37 \mathrm{E}-07$ \\
\hline 3 to 4 & $4.88 \mathrm{E}-05$ & $5.06 \mathrm{E}-05$ & $4.95 \mathrm{E}-05$ & $8.48 \mathrm{E}-07$ & 8.63E-07 & $8.59 \mathrm{E}-07$ & 3.37E-07 & $3.37 \mathrm{E}-07$ & $3.37 \mathrm{E}-07$ \\
\hline 4 to 5 & 4.32E-05 & 4.30E-05 & $4.31 \mathrm{E}-05$ & $8.26 \mathrm{E}-07$ & $8.39 \mathrm{E}-07$ & $8.24 \mathrm{E}-07$ & 3.37E-07 & 3.37E-07 & 3.37E-07 \\
\hline 5 to 6 & $3.91 \mathrm{E}-05$ & $3.95 \mathrm{E}-05$ & $3.93 \mathrm{E}-05$ & $7.57 \mathrm{E}-07$ & 8.11E-07 & 8.04E-07 & 3.00E-07 & $3.37 \mathrm{E}-07$ & $3.37 \mathrm{E}-07$ \\
\hline 6 to 9 & $3.95 \mathrm{E}-05$ & $4.08 \mathrm{E}-05$ & $4.01 \mathrm{E}-05$ & $7.30 \mathrm{E}-07$ & 7.90E-07 & $7.36 \mathrm{E}-07$ & 3.37E-07 & $3.76 \mathrm{E}-07$ & 3.37E-07 \\
\hline 9 to 12 & $3.08 \mathrm{E}-05$ & $3.17 \mathrm{E}-05$ & $3.11 \mathrm{E}-05$ & $6.87 \mathrm{E}-07$ & 7.43E-07 & $6.94 \mathrm{E}-07$ & $3.76 \mathrm{E}-07$ & 4.13E-07 & $3.76 \mathrm{E}-07$ \\
\hline 12 to 15 & $2.59 \mathrm{E}-05$ & $2.65 \mathrm{E}-05$ & $2.62 \mathrm{E}-05$ & $6.63 \mathrm{E}-07$ & 7.41E-07 & $7.03 \mathrm{E}-07$ & $4.13 \mathrm{E}-07$ & 4.87E-07 & $4.50 \mathrm{E}-07$ \\
\hline 15 to 18 & $2.35 \mathrm{E}-05$ & $2.26 \mathrm{E}-05$ & $2.31 \mathrm{E}-05$ & $5.71 \mathrm{E}-07$ & $6.83 \mathrm{E}-07$ & $6.10 \mathrm{E}-07$ & $3.37 \mathrm{E}-07$ & $4.50 \mathrm{E}-07$ & $3.76 \mathrm{E}-07$ \\
\hline 18 to 45 & $9.13 \mathrm{E}-06$ & $9.41 \mathrm{E}-06$ & $9.31 \mathrm{E}-06$ & $1.61 \mathrm{E}-07$ & 1.67E-07 & $1.65 \mathrm{E}-07$ & $9.97 \mathrm{E}-08$ & $9.97 \mathrm{E}-08$ & 9.97E-08 \\
\hline 45 to 60 & $9.15 \mathrm{E}-06$ & $8.78 \mathrm{E}-06$ & $9.09 \mathrm{E}-06$ & $1.49 \mathrm{E}-07$ & $1.56 \mathrm{E}-07$ & $1.48 \mathrm{E}-07$ & $8.75 \mathrm{E}-08$ & $8.75 \mathrm{E}-08$ & $8.75 \mathrm{E}-08$ \\
\hline 60 to 80 & $9.32 \mathrm{E}-06$ & $8.70 \mathrm{E}-06$ & $9.04 \mathrm{E}-06$ & $1.38 \mathrm{E}-07$ & $1.64 \mathrm{E}-07$ & $1.44 \mathrm{E}-07$ & 7.50E-08 & $7.50 \mathrm{E}-08$ & $7.50 \mathrm{E}-08$ \\
\hline$\geq 80$ & $9.09 \mathrm{E}-06$ & 8.04E-06 & $8.38 \mathrm{E}-06$ & $1.32 \mathrm{E}-07$ & $1.34 \mathrm{E}-07$ & $1.35 \mathrm{E}-07$ & $6.25 \mathrm{E}-08$ & $6.25 \mathrm{E}-08$ & $6.25 \mathrm{E}-08$ \\
\hline
\end{tabular}


Table S15. Estimated lifetime cancer risk (ELCR) of NDPA by age groups and sex under reasonable maximum exposure.

\begin{tabular}{|c|c|c|c|c|c|c|c|c|c|}
\hline \multirow{2}{*}{$\begin{array}{l}\text { Age Group } \\
\text { (yeas) }\end{array}$} & \multicolumn{3}{|c|}{ ELCR $_{\text {oral }}$} & \multicolumn{3}{|c|}{ ELCR $_{\text {dermal }}$} & \multicolumn{3}{|c|}{$\mathbf{E C L R}_{\text {inhalation }}$} \\
\hline & Male & Female & Total & Male & Female & Total & Male & Female & Total \\
\hline Birth to 0.25 & $3.07 \mathrm{E}-04$ & 3.84E-04 & $3.36 \mathrm{E}-04$ & $6.75 \mathrm{E}-06$ & 7.13E-06 & 7.07E-06 & $2.40 \mathrm{E}-06$ & $2.26 \mathrm{E}-06$ & $2.40 \mathrm{E}-06$ \\
\hline 0.25 to 0.5 & $5.40 \mathrm{E}-04$ & 5.03E-04 & $5.44 \mathrm{E}-04$ & $6.21 \mathrm{E}-06$ & $6.65 \mathrm{E}-06$ & $6.39 \mathrm{E}-06$ & $2.40 \mathrm{E}-06$ & $2.55 \mathrm{E}-06$ & $2.40 \mathrm{E}-06$ \\
\hline 0.5 to 0.75 & $6.28 \mathrm{E}-04$ & $6.69 \mathrm{E}-04$ & $6.40 \mathrm{E}-04$ & $6.41 \mathrm{E}-06$ & $6.59 \mathrm{E}-06$ & $6.55 \mathrm{E}-06$ & $2.70 \mathrm{E}-06$ & $2.86 \mathrm{E}-06$ & $2.86 \mathrm{E}-06$ \\
\hline 0.75 to 1 & $8.95 \mathrm{E}-04$ & $6.60 \mathrm{E}-04$ & $8.23 \mathrm{E}-04$ & $6.41 \mathrm{E}-06$ & $6.56 \mathrm{E}-06$ & $6.48 \mathrm{E}-06$ & $3.01 \mathrm{E}-06$ & $3.01 \mathrm{E}-06$ & $3.01 \mathrm{E}-06$ \\
\hline 1 to 2 & 7.01E-04 & $6.63 \mathrm{E}-04$ & 6.79E-04 & $6.18 \mathrm{E}-06$ & $6.37 \mathrm{E}-06$ & $6.25 \mathrm{E}-06$ & $2.86 \mathrm{E}-06$ & $2.86 \mathrm{E}-06$ & $2.86 \mathrm{E}-06$ \\
\hline 2 to 3 & $1.29 \mathrm{E}-04$ & $1.41 \mathrm{E}-04$ & $1.33 \mathrm{E}-04$ & $1.78 \mathrm{E}-06$ & $1.83 \mathrm{E}-06$ & $1.81 \mathrm{E}-06$ & $8.56 \mathrm{E}-07$ & $8.56 \mathrm{E}-07$ & $8.56 \mathrm{E}-07$ \\
\hline 3 to 4 & 1.19E-04 & $1.23 \mathrm{E}-04$ & $1.21 \mathrm{E}-04$ & 1.69E-06 & $1.77 \mathrm{E}-06$ & $1.75 \mathrm{E}-06$ & $8.11 \mathrm{E}-07$ & $9.04 \mathrm{E}-07$ & $8.56 \mathrm{E}-07$ \\
\hline 4 to 5 & $1.05 \mathrm{E}-04$ & $1.08 \mathrm{E}-04$ & $1.07 \mathrm{E}-04$ & $1.75 \mathrm{E}-06$ & $1.72 \mathrm{E}-06$ & $1.71 \mathrm{E}-06$ & $9.04 \mathrm{E}-07$ & $8.56 \mathrm{E}-07$ & $8.56 \mathrm{E}-07$ \\
\hline 5 to 6 & $9.49 \mathrm{E}-05$ & $9.76 \mathrm{E}-05$ & $9.58 \mathrm{E}-05$ & $1.60 \mathrm{E}-06$ & $1.68 \mathrm{E}-06$ & $1.62 \mathrm{E}-06$ & $8.11 \mathrm{E}-07$ & $8.56 \mathrm{E}-07$ & $8.11 \mathrm{E}-07$ \\
\hline 6 to 9 & $8.47 \mathrm{E}-05$ & $9.04 \mathrm{E}-05$ & $8.72 \mathrm{E}-05$ & 1.64E-06 & $1.66 \mathrm{E}-06$ & $1.66 \mathrm{E}-06$ & $9.04 \mathrm{E}-07$ & $9.04 \mathrm{E}-07$ & $9.04 \mathrm{E}-07$ \\
\hline 9 to 12 & $6.54 \mathrm{E}-05$ & $6.88 \mathrm{E}-05$ & $6.71 \mathrm{E}-05$ & $1.54 \mathrm{E}-06$ & $1.63 \mathrm{E}-06$ & $1.59 \mathrm{E}-06$ & $9.49 \mathrm{E}-07$ & $1.04 \mathrm{E}-06$ & $9.94 \mathrm{E}-07$ \\
\hline 12 to 15 & $5.96 \mathrm{E}-05$ & $6.32 \mathrm{E}-05$ & $6.13 \mathrm{E}-05$ & $1.48 \mathrm{E}-06$ & $1.61 \mathrm{E}-06$ & $1.58 \mathrm{E}-06$ & $1.08 \mathrm{E}-06$ & $1.35 \mathrm{E}-06$ & $1.26 \mathrm{E}-06$ \\
\hline 15 to 18 & $6.31 \mathrm{E}-05$ & $6.47 \mathrm{E}-05$ & $6.38 \mathrm{E}-05$ & $1.29 \mathrm{E}-06$ & $1.46 \mathrm{E}-06$ & $1.40 \mathrm{E}-06$ & $1.04 \mathrm{E}-06$ & $1.31 \mathrm{E}-06$ & $1.17 \mathrm{E}-06$ \\
\hline 18 to 45 & $2.97 \mathrm{E}-05$ & $3.16 \mathrm{E}-05$ & 3.13E-05 & $3.42 \mathrm{E}-07$ & $3.80 \mathrm{E}-07$ & $3.62 \mathrm{E}-07$ & $2.70 \mathrm{E}-07$ & $3.01 \mathrm{E}-07$ & $2.86 \mathrm{E}-07$ \\
\hline 45 to 60 & $3.10 \mathrm{E}-05$ & 2.92E-05 & $3.07 \mathrm{E}-05$ & $3.28 \mathrm{E}-07$ & $3.37 \mathrm{E}-07$ & $3.40 \mathrm{E}-07$ & $2.55 \mathrm{E}-07$ & $2.70 \mathrm{E}-07$ & $2.70 \mathrm{E}-07$ \\
\hline 60 to 80 & $3.02 \mathrm{E}-05$ & $2.78 \mathrm{E}-05$ & $2.93 \mathrm{E}-05$ & $3.10 \mathrm{E}-07$ & $3.35 \mathrm{E}-07$ & $3.26 \mathrm{E}-07$ & $2.26 \mathrm{E}-07$ & $2.40 \mathrm{E}-07$ & $2.26 \mathrm{E}-07$ \\
\hline$\geq 80$ & $3.03 \mathrm{E}-05$ & $2.77 \mathrm{E}-05$ & $2.97 \mathrm{E}-05$ & $3.59 \mathrm{E}-07$ & $3.39 \mathrm{E}-07$ & $3.49 \mathrm{E}-07$ & $2.70 \mathrm{E}-07$ & $2.26 \mathrm{E}-07$ & $2.40 \mathrm{E}-07$ \\
\hline
\end{tabular}


Table S16. Estimated lifetime cancer risk (ELCR) of NPYR by age groups and sex under central tendency exposure.

\begin{tabular}{|c|c|c|c|c|c|c|c|c|c|}
\hline \multirow{2}{*}{$\begin{array}{l}\text { Age Group } \\
\text { (yeas) }\end{array}$} & \multicolumn{3}{|c|}{ ELCR $_{\text {oral }}$} & \multicolumn{3}{|c|}{ ELCR $_{\text {dermal }}$} & \multicolumn{3}{|c|}{$\mathbf{E C L R}_{\text {inhalation }}$} \\
\hline & Male & Female & Total & Male & Female & Total & Male & Female & Total \\
\hline Birth to 0.25 & $3.58 \mathrm{E}-05$ & $3.52 \mathrm{E}-05$ & $3.53 \mathrm{E}-05$ & $1.07 \mathrm{E}-07$ & $9.82 \mathrm{E}-08$ & $1.06 \mathrm{E}-07$ & $2.41 \mathrm{E}-09$ & $1.93 \mathrm{E}-09$ & 2.41E-09 \\
\hline 0.25 to 0.5 & $5.56 \mathrm{E}-05$ & $5.17 \mathrm{E}-05$ & $5.42 \mathrm{E}-05$ & $1.16 \mathrm{E}-07$ & $1.23 \mathrm{E}-07$ & $1.20 \mathrm{E}-07$ & 3.36E-09 & 3.36E-09 & 3.36E-09 \\
\hline 0.5 to 0.75 & 7.86E-05 & 8.39E-05 & $8.08 \mathrm{E}-05$ & $1.15 \mathrm{E}-07$ & $1.25 \mathrm{E}-07$ & $1.25 \mathrm{E}-07$ & 3.36E-09 & $3.85 \mathrm{E}-09$ & $3.85 \mathrm{E}-09$ \\
\hline 0.75 to 1 & $1.03 \mathrm{E}-04$ & $1.02 \mathrm{E}-04$ & $1.03 \mathrm{E}-04$ & $1.20 \mathrm{E}-07$ & $1.30 \mathrm{E}-07$ & $1.21 \mathrm{E}-07$ & 3.85E-09 & 4.33E-09 & $3.85 \mathrm{E}-09$ \\
\hline 1 to 2 & $1.01 \mathrm{E}-04$ & $1.01 \mathrm{E}-04$ & $1.01 \mathrm{E}-04$ & $1.16 \mathrm{E}-07$ & $1.20 \mathrm{E}-07$ & $1.18 \mathrm{E}-07$ & $3.85 \mathrm{E}-09$ & $3.85 \mathrm{E}-09$ & $3.85 \mathrm{E}-09$ \\
\hline 2 to 3 & $2.18 \mathrm{E}-05$ & 2.31E-05 & $2.23 \mathrm{E}-05$ & $3.58 \mathrm{E}-08$ & $3.66 \mathrm{E}-08$ & 3.64E-08 & $1.30 \mathrm{E}-09$ & $1.30 \mathrm{E}-09$ & $1.30 \mathrm{E}-09$ \\
\hline 3 to 4 & 2.04E-05 & $2.10 \mathrm{E}-05$ & $2.06 \mathrm{E}-05$ & $3.46 \mathrm{E}-08$ & $3.52 \mathrm{E}-08$ & $3.50 \mathrm{E}-08$ & $1.30 \mathrm{E}-09$ & $1.30 \mathrm{E}-09$ & $1.30 \mathrm{E}-09$ \\
\hline 4 to 5 & $1.80 \mathrm{E}-05$ & $1.78 \mathrm{E}-05$ & $1.80 \mathrm{E}-05$ & $3.36 \mathrm{E}-08$ & $3.42 \mathrm{E}-08$ & 3.36E-08 & $1.30 \mathrm{E}-09$ & $1.30 \mathrm{E}-09$ & $1.30 \mathrm{E}-09$ \\
\hline 5 to 6 & $1.63 \mathrm{E}-05$ & $1.64 \mathrm{E}-05$ & $1.63 \mathrm{E}-05$ & $3.08 \mathrm{E}-08$ & $3.31 \mathrm{E}-08$ & $3.28 \mathrm{E}-08$ & $1.15 \mathrm{E}-09$ & $1.30 \mathrm{E}-09$ & $1.30 \mathrm{E}-09$ \\
\hline 6 to 9 & $1.64 \mathrm{E}-05$ & $1.70 \mathrm{E}-05$ & $1.67 \mathrm{E}-05$ & 2.97E-08 & $3.22 \mathrm{E}-08$ & $3.00 \mathrm{E}-08$ & $1.30 \mathrm{E}-09$ & $1.45 \mathrm{E}-09$ & $1.30 \mathrm{E}-09$ \\
\hline 9 to 12 & $1.28 \mathrm{E}-05$ & $1.32 \mathrm{E}-05$ & $1.30 \mathrm{E}-05$ & $2.80 \mathrm{E}-08$ & 3.03E-08 & 2.83E-08 & $1.45 \mathrm{E}-09$ & $1.59 \mathrm{E}-09$ & $1.45 \mathrm{E}-09$ \\
\hline 12 to 15 & $1.08 \mathrm{E}-05$ & $1.10 \mathrm{E}-05$ & $1.09 \mathrm{E}-05$ & $2.70 \mathrm{E}-08$ & 3.03E-08 & $2.86 \mathrm{E}-08$ & $1.59 \mathrm{E}-09$ & $1.87 \mathrm{E}-09$ & 1.73E-09 \\
\hline 15 to 18 & $9.78 \mathrm{E}-06$ & $9.37 \mathrm{E}-06$ & $9.61 \mathrm{E}-06$ & 2.33E-08 & 2.79E-08 & 2.49E-08 & $1.30 \mathrm{E}-09$ & $1.73 \mathrm{E}-09$ & $1.45 \mathrm{E}-09$ \\
\hline 18 to 45 & $3.80 \mathrm{E}-06$ & 3.91E-06 & $3.87 \mathrm{E}-06$ & $6.57 \mathrm{E}-09$ & 6.81E-09 & 6.73E-09 & $3.85 \mathrm{E}-10$ & $3.85 \mathrm{E}-10$ & $3.85 \mathrm{E}-10$ \\
\hline 45 to 60 & $3.81 \mathrm{E}-06$ & $3.66 \mathrm{E}-06$ & $3.78 \mathrm{E}-06$ & $6.08 \mathrm{E}-09$ & 6.36E-09 & $6.05 \mathrm{E}-09$ & $3.36 \mathrm{E}-10$ & $3.36 \mathrm{E}-10$ & $3.36 \mathrm{E}-10$ \\
\hline 60 to 80 & $3.87 \mathrm{E}-06$ & $3.62 \mathrm{E}-06$ & $3.77 \mathrm{E}-06$ & 5.61E-09 & 6.69E-09 & 5.89E-09 & $2.88 \mathrm{E}-10$ & $2.88 \mathrm{E}-10$ & $2.88 \mathrm{E}-10$ \\
\hline$\geq 80$ & $3.78 \mathrm{E}-06$ & 3.35E-06 & $3.48 \mathrm{E}-06$ & $5.41 \mathrm{E}-09$ & $5.47 \mathrm{E}-09$ & 5.52E-09 & $2.41 \mathrm{E}-10$ & $2.41 \mathrm{E}-10$ & $2.41 \mathrm{E}-10$ \\
\hline
\end{tabular}


Table S17. Estimated lifetime cancer risk (ELCR) of NPYR by age groups and sex under reasonable maximum exposure.

\begin{tabular}{|c|c|c|c|c|c|c|c|c|c|}
\hline \multirow{2}{*}{$\begin{array}{l}\text { Age Group } \\
\text { (yeas) }\end{array}$} & \multicolumn{3}{|c|}{ ELCR $_{\text {oral }}$} & \multicolumn{3}{|c|}{$\mathbf{E L C R}_{\text {dermal }}$} & \multicolumn{3}{|c|}{$\mathbf{E C L R}_{\text {inhalation }}$} \\
\hline & Male & Female & Total & Male & Female & Total & Male & Female & Total \\
\hline Birth to 0.25 & $1.12 \mathrm{E}-04$ & $1.40 \mathrm{E}-04$ & $1.22 \mathrm{E}-04$ & $2.40 \mathrm{E}-07$ & $2.54 \mathrm{E}-07$ & $2.52 \mathrm{E}-07$ & $8.05 \mathrm{E}-09$ & $7.56 \mathrm{E}-09$ & 8.05E-09 \\
\hline 0.25 to 0.5 & $1.96 \mathrm{E}-04$ & $1.83 \mathrm{E}-04$ & $1.97 \mathrm{E}-04$ & $2.21 \mathrm{E}-07$ & $2.36 \mathrm{E}-07$ & $2.27 \mathrm{E}-07$ & $8.05 \mathrm{E}-09$ & 8.57E-09 & $8.05 E-09$ \\
\hline 0.5 to 0.75 & $2.29 \mathrm{E}-04$ & $2.43 \mathrm{E}-04$ & $2.32 \mathrm{E}-04$ & $2.27 \mathrm{E}-07$ & $2.34 \mathrm{E}-07$ & $2.33 \mathrm{E}-07$ & $9.06 \mathrm{E}-09$ & $9.57 \mathrm{E}-09$ & $9.57 \mathrm{E}-09$ \\
\hline 0.75 to 1 & $3.25 \mathrm{E}-04$ & $2.40 \mathrm{E}-04$ & $2.98 \mathrm{E}-04$ & $2.27 \mathrm{E}-07$ & $2.33 \mathrm{E}-07$ & $2.31 \mathrm{E}-07$ & $1.01 \mathrm{E}-08$ & $1.01 \mathrm{E}-08$ & $1.01 \mathrm{E}-08$ \\
\hline 1 to 2 & $2.54 \mathrm{E}-04$ & $2.41 \mathrm{E}-04$ & $2.46 \mathrm{E}-04$ & $2.20 \mathrm{E}-07$ & $2.26 \mathrm{E}-07$ & $2.22 \mathrm{E}-07$ & $9.57 \mathrm{E}-09$ & $9.57 \mathrm{E}-09$ & 9.57E-09 \\
\hline 2 to 3 & $4.68 \mathrm{E}-05$ & $5.14 \mathrm{E}-05$ & $4.84 \mathrm{E}-05$ & $6.34 \mathrm{E}-08$ & $6.49 \mathrm{E}-08$ & $6.43 \mathrm{E}-08$ & $2.87 \mathrm{E}-09$ & $2.87 \mathrm{E}-09$ & 2.87E-09 \\
\hline 3 to 4 & 4.32E-05 & $4.46 \mathrm{E}-05$ & 4.37E-05 & $6.02 \mathrm{E}-08$ & $6.31 \mathrm{E}-08$ & 6.19E-08 & 2.72E-09 & $3.02 \mathrm{E}-09$ & 2.87E-09 \\
\hline 4 to 5 & $3.82 \mathrm{E}-05$ & $3.93 \mathrm{E}-05$ & $3.86 \mathrm{E}-05$ & $6.24 \mathrm{E}-08$ & $6.11 \mathrm{E}-08$ & $6.08 \mathrm{E}-08$ & $3.02 \mathrm{E}-09$ & $2.87 \mathrm{E}-09$ & 2.87E-09 \\
\hline 5 to 6 & $3.43 \mathrm{E}-05$ & $3.55 \mathrm{E}-05$ & $3.48 \mathrm{E}-05$ & $5.68 \mathrm{E}-08$ & 5.99E-08 & $5.77 \mathrm{E}-08$ & 2.72E-09 & 2.87E-09 & 2.72E-09 \\
\hline 6 to 9 & $3.07 \mathrm{E}-05$ & $3.28 \mathrm{E}-05$ & $3.16 \mathrm{E}-05$ & $5.84 \mathrm{E}-08$ & $5.90 \mathrm{E}-08$ & 5.91E-08 & $3.02 \mathrm{E}-09$ & $3.02 \mathrm{E}-09$ & $3.02 \mathrm{E}-09$ \\
\hline 9 to 12 & $2.37 \mathrm{E}-05$ & $2.50 \mathrm{E}-05$ & $2.44 \mathrm{E}-05$ & $5.48 \mathrm{E}-08$ & $5.80 \mathrm{E}-08$ & $5.65 \mathrm{E}-08$ & $3.17 \mathrm{E}-09$ & $3.47 \mathrm{E}-09$ & 3.33E-09 \\
\hline 12 to 15 & $2.16 \mathrm{E}-05$ & $2.30 \mathrm{E}-05$ & $2.23 \mathrm{E}-05$ & $5.25 \mathrm{E}-08$ & $5.71 \mathrm{E}-08$ & $5.61 \mathrm{E}-08$ & $3.63 \mathrm{E}-09$ & $4.54 \mathrm{E}-09$ & 4.23E-09 \\
\hline 15 to 18 & 2.29E-05 & $2.35 \mathrm{E}-05$ & 2.32E-05 & $4.57 \mathrm{E}-08$ & $5.18 \mathrm{E}-08$ & $4.96 \mathrm{E}-08$ & $3.47 \mathrm{E}-09$ & 4.38E-09 & $3.93 \mathrm{E}-09$ \\
\hline 18 to 45 & $1.08 \mathrm{E}-05$ & $1.14 \mathrm{E}-05$ & $1.13 \mathrm{E}-05$ & $1.22 \mathrm{E}-08$ & $1.35 \mathrm{E}-08$ & $1.29 \mathrm{E}-08$ & $9.06 \mathrm{E}-10$ & $1.01 \mathrm{E}-09$ & $9.57 \mathrm{E}-10$ \\
\hline 45 to 60 & $1.12 \mathrm{E}-05$ & $1.06 \mathrm{E}-05$ & $1.11 \mathrm{E}-05$ & $1.16 \mathrm{E}-08$ & $1.20 \mathrm{E}-08$ & $1.21 \mathrm{E}-08$ & $8.57 \mathrm{E}-10$ & $9.06 \mathrm{E}-10$ & $9.06 \mathrm{E}-10$ \\
\hline 60 to 80 & $1.09 \mathrm{E}-05$ & $1.01 \mathrm{E}-05$ & $1.06 \mathrm{E}-05$ & $1.10 \mathrm{E}-08$ & 1.19E-08 & $1.15 \mathrm{E}-08$ & $7.56 \mathrm{E}-10$ & $8.05 \mathrm{E}-10$ & $7.56 \mathrm{E}-10$ \\
\hline$\geq 80$ & $1.10 \mathrm{E}-05$ & $1.00 \mathrm{E}-05$ & $1.08 \mathrm{E}-05$ & $1.28 \mathrm{E}-08$ & $1.21 \mathrm{E}-08$ & $1.24 \mathrm{E}-08$ & $9.06 \mathrm{E}-10$ & $7.56 \mathrm{E}-10$ & $8.05 \mathrm{E}-10$ \\
\hline
\end{tabular}


Table S18. Estimated lifetime cancer risk (ELCR) of NMEA by age groups and sex under central tendency exposure.

\begin{tabular}{|c|c|c|c|c|c|c|c|c|c|}
\hline \multirow{2}{*}{$\begin{array}{l}\text { Age Group } \\
\text { (yeas) }\end{array}$} & \multicolumn{3}{|c|}{ ELCR $_{\text {oral }}$} & \multicolumn{3}{|c|}{ ELCR $_{\text {dermal }}$} & \multicolumn{3}{|c|}{ ECLR $_{\text {inhalation }}$} \\
\hline & Male & Female & Total & Male & Female & Total & Male & Female & Total \\
\hline Birth to 0.25 & $1.70 \mathrm{E}-04$ & $1.66 \mathrm{E}-04$ & $1.67 \mathrm{E}-04$ & $7.84 \mathrm{E}-07$ & 7.17E-07 & $7.75 \mathrm{E}-07$ & $3.50 \mathrm{E}-07$ & $2.79 \mathrm{E}-07$ & $3.50 \mathrm{E}-07$ \\
\hline 0.25 to 0.5 & $2.64 \mathrm{E}-04$ & 2.44E-04 & $2.57 \mathrm{E}-04$ & $8.53 \mathrm{E}-07$ & 8.97E-07 & 8.74E-07 & $4.89 \mathrm{E}-07$ & 4.89E-07 & 4.89E-07 \\
\hline 0.5 to 0.75 & $3.72 \mathrm{E}-04$ & 3.97E-04 & $3.82 \mathrm{E}-04$ & $8.44 \mathrm{E}-07$ & $9.12 \mathrm{E}-07$ & $9.12 \mathrm{E}-07$ & $4.89 \mathrm{E}-07$ & $5.60 \mathrm{E}-07$ & $5.60 \mathrm{E}-07$ \\
\hline 0.75 to 1 & 4.89E-04 & $4.86 \mathrm{E}-04$ & $4.88 \mathrm{E}-04$ & 8.77E-07 & $9.47 \mathrm{E}-07$ & 8.84E-07 & $5.60 \mathrm{E}-07$ & $6.30 \mathrm{E}-07$ & $5.60 \mathrm{E}-07$ \\
\hline 1 to 2 & 4.79E-04 & $4.81 \mathrm{E}-04$ & $4.78 \mathrm{E}-04$ & $8.50 \mathrm{E}-07$ & 8.70E-07 & $8.57 \mathrm{E}-07$ & $5.60 \mathrm{E}-07$ & $5.60 \mathrm{E}-07$ & $5.60 \mathrm{E}-07$ \\
\hline 2 to 3 & $1.03 \mathrm{E}-04$ & $1.09 \mathrm{E}-04$ & $1.06 \mathrm{E}-04$ & 2.62E-07 & 2.67E-07 & $2.65 \mathrm{E}-07$ & $1.89 \mathrm{E}-07$ & $1.89 \mathrm{E}-07$ & $1.89 \mathrm{E}-07$ \\
\hline 3 to 4 & $9.62 \mathrm{E}-05$ & $9.98 \mathrm{E}-05$ & $9.76 \mathrm{E}-05$ & $2.53 \mathrm{E}-07$ & $2.57 \mathrm{E}-07$ & $2.55 \mathrm{E}-07$ & $1.89 \mathrm{E}-07$ & $1.89 \mathrm{E}-07$ & $1.89 \mathrm{E}-07$ \\
\hline 4 to 5 & $8.52 \mathrm{E}-05$ & $8.48 \mathrm{E}-05$ & $8.48 \mathrm{E}-05$ & $2.47 \mathrm{E}-07$ & $2.49 \mathrm{E}-07$ & $2.45 \mathrm{E}-07$ & $1.89 \mathrm{E}-07$ & $1.89 \mathrm{E}-07$ & $1.89 \mathrm{E}-07$ \\
\hline 5 to 6 & $7.71 \mathrm{E}-05$ & 7.79E-05 & $7.75 \mathrm{E}-05$ & $2.25 \mathrm{E}-07$ & 2.42E-07 & 2.39E-07 & $1.67 \mathrm{E}-07$ & $1.89 \mathrm{E}-07$ & $1.89 \mathrm{E}-07$ \\
\hline 6 to 9 & $7.80 \mathrm{E}-05$ & $8.04 \mathrm{E}-05$ & $7.90 \mathrm{E}-05$ & $2.18 \mathrm{E}-07$ & $2.35 \mathrm{E}-07$ & 2.19E-07 & $1.89 \mathrm{E}-07$ & $2.10 \mathrm{E}-07$ & $1.89 \mathrm{E}-07$ \\
\hline 9 to 12 & $6.06 \mathrm{E}-05$ & $6.25 \mathrm{E}-05$ & $6.14 \mathrm{E}-05$ & $2.05 \mathrm{E}-07$ & $2.21 \mathrm{E}-07$ & $2.06 \mathrm{E}-07$ & $2.10 \mathrm{E}-07$ & $2.31 \mathrm{E}-07$ & $2.10 \mathrm{E}-07$ \\
\hline 12 to 15 & 5.09E-05 & $5.22 \mathrm{E}-05$ & $5.16 \mathrm{E}-05$ & $1.97 \mathrm{E}-07$ & 2.20E-07 & 2.09E-07 & $2.31 \mathrm{E}-07$ & $2.73 \mathrm{E}-07$ & $2.52 \mathrm{E}-07$ \\
\hline 15 to 18 & 4.64E-05 & 4.44E-05 & $4.55 \mathrm{E}-05$ & $1.70 \mathrm{E}-07$ & 2.04E-07 & $1.81 \mathrm{E}-07$ & $1.89 \mathrm{E}-07$ & $2.52 \mathrm{E}-07$ & $2.10 \mathrm{E}-07$ \\
\hline 18 to 45 & $1.80 \mathrm{E}-05$ & $1.85 \mathrm{E}-05$ & $1.84 \mathrm{E}-05$ & $4.81 \mathrm{E}-08$ & 4.98E-08 & 4.91E-08 & $5.60 \mathrm{E}-08$ & $5.60 \mathrm{E}-08$ & $5.60 \mathrm{E}-08$ \\
\hline 45 to 60 & $1.80 \mathrm{E}-05$ & $1.74 \mathrm{E}-05$ & $1.79 \mathrm{E}-05$ & 4.44E-08 & 4.64E-08 & 4.43E-08 & $4.89 \mathrm{E}-08$ & 4.89E-08 & $4.89 \mathrm{E}-08$ \\
\hline 60 to 80 & $1.84 \mathrm{E}-05$ & $1.71 \mathrm{E}-05$ & $1.79 \mathrm{E}-05$ & 4.10E-08 & $4.89 \mathrm{E}-08$ & 4.30E-08 & 4.20E-08 & $4.20 \mathrm{E}-08$ & $4.20 \mathrm{E}-08$ \\
\hline$\geq 80$ & $1.79 \mathrm{E}-05$ & $1.58 \mathrm{E}-05$ & $1.65 \mathrm{E}-05$ & $3.95 \mathrm{E}-08$ & $4.00 \mathrm{E}-08$ & $4.03 \mathrm{E}-08$ & $3.50 \mathrm{E}-08$ & $3.50 \mathrm{E}-08$ & $3.50 \mathrm{E}-08$ \\
\hline
\end{tabular}


Table S19. Estimated lifetime cancer risk (ELCR) of NMEA by age groups and sex under reasonable maximum exposure.

\begin{tabular}{|c|c|c|c|c|c|c|c|c|c|}
\hline \multirow{2}{*}{$\begin{array}{l}\text { Age Group } \\
\text { (yeas) }\end{array}$} & \multicolumn{3}{|c|}{ ELCR $_{\text {oral }}$} & \multicolumn{3}{|c|}{ ELCR $_{\text {dermal }}$} & \multicolumn{3}{|c|}{$\mathbf{E C L R}_{\text {inhalation }}$} \\
\hline & Male & Female & Total & Male & Female & Total & Male & Female & Total \\
\hline Birth to 0.25 & $6.85 \mathrm{E}-04$ & $8.56 \mathrm{E}-04$ & 7.49E-04 & $2.27 \mathrm{E}-06$ & $2.40 \mathrm{E}-06$ & $2.38 \mathrm{E}-06$ & $1.52 \mathrm{E}-06$ & $1.43 \mathrm{E}-06$ & $1.52 \mathrm{E}-06$ \\
\hline 0.25 to 0.5 & $1.20 \mathrm{E}-03$ & $1.12 \mathrm{E}-03$ & $1.21 \mathrm{E}-03$ & $2.09 \mathrm{E}-06$ & $2.24 \mathrm{E}-06$ & $2.15 \mathrm{E}-06$ & $1.52 \mathrm{E}-06$ & $1.62 \mathrm{E}-06$ & $1.52 \mathrm{E}-06$ \\
\hline 0.5 to 0.75 & $1.41 \mathrm{E}-03$ & $1.50 \mathrm{E}-03$ & $1.43 \mathrm{E}-03$ & $2.16 \mathrm{E}-06$ & $2.22 \mathrm{E}-06$ & $2.20 \mathrm{E}-06$ & $1.71 \mathrm{E}-06$ & $1.80 \mathrm{E}-06$ & $1.80 \mathrm{E}-06$ \\
\hline 0.75 to 1 & $1.99 \mathrm{E}-03$ & $1.47 \mathrm{E}-03$ & $1.83 \mathrm{E}-03$ & $2.16 \mathrm{E}-06$ & $2.20 \mathrm{E}-06$ & $2.18 \mathrm{E}-06$ & $1.90 \mathrm{E}-06$ & $1.90 \mathrm{E}-06$ & $1.90 \mathrm{E}-06$ \\
\hline 1 to 2 & $1.56 \mathrm{E}-03$ & $1.47 \mathrm{E}-03$ & $1.52 \mathrm{E}-03$ & $2.08 \mathrm{E}-06$ & $2.15 \mathrm{E}-06$ & $2.10 \mathrm{E}-06$ & $1.80 \mathrm{E}-06$ & $1.80 \mathrm{E}-06$ & $1.80 \mathrm{E}-06$ \\
\hline 2 to 3 & 2.87E-04 & $3.16 \mathrm{E}-04$ & $2.97 \mathrm{E}-04$ & $6.01 \mathrm{E}-07$ & $6.14 \mathrm{E}-07$ & $6.08 \mathrm{E}-07$ & $5.41 \mathrm{E}-07$ & $5.41 \mathrm{E}-07$ & $5.41 \mathrm{E}-07$ \\
\hline 3 to 4 & $2.65 \mathrm{E}-04$ & $2.74 \mathrm{E}-04$ & $2.69 \mathrm{E}-04$ & $5.70 \mathrm{E}-07$ & $5.98 \mathrm{E}-07$ & $5.86 \mathrm{E}-07$ & $5.13 \mathrm{E}-07$ & $5.70 \mathrm{E}-07$ & $5.41 \mathrm{E}-07$ \\
\hline 4 to 5 & 2.34E-04 & $2.41 \mathrm{E}-04$ & $2.37 \mathrm{E}-04$ & $5.90 \mathrm{E}-07$ & $5.79 \mathrm{E}-07$ & $5.76 \mathrm{E}-07$ & $5.70 \mathrm{E}-07$ & $5.41 \mathrm{E}-07$ & $5.41 \mathrm{E}-07$ \\
\hline 5 to 6 & $2.10 \mathrm{E}-04$ & $2.18 \mathrm{E}-04$ & $2.14 \mathrm{E}-04$ & $5.38 \mathrm{E}-07$ & $5.67 \mathrm{E}-07$ & 5.47E-07 & $5.13 \mathrm{E}-07$ & $5.41 \mathrm{E}-07$ & 5.13E-07 \\
\hline 6 to 9 & $1.89 \mathrm{E}-04$ & $2.02 \mathrm{E}-04$ & $1.95 \mathrm{E}-04$ & $5.52 \mathrm{E}-07$ & $5.59 \mathrm{E}-07$ & $5.60 \mathrm{E}-07$ & $5.70 \mathrm{E}-07$ & $5.70 \mathrm{E}-07$ & $5.70 \mathrm{E}-07$ \\
\hline 9 to 12 & $1.46 \mathrm{E}-04$ & $1.53 \mathrm{E}-04$ & $1.50 \mathrm{E}-04$ & $5.18 \mathrm{E}-07$ & $5.49 \mathrm{E}-07$ & 5.35E-07 & $5.98 \mathrm{E}-07$ & $6.56 \mathrm{E}-07$ & $6.28 \mathrm{E}-07$ \\
\hline 12 to 15 & $1.33 \mathrm{E}-04$ & $1.41 \mathrm{E}-04$ & $1.37 \mathrm{E}-04$ & 4.97E-07 & $5.41 \mathrm{E}-07$ & $5.32 \mathrm{E}-07$ & $6.84 \mathrm{E}-07$ & $8.55 \mathrm{E}-07$ & 7.98E-07 \\
\hline 15 to 18 & $1.41 \mathrm{E}-04$ & $1.44 \mathrm{E}-04$ & $1.42 \mathrm{E}-04$ & $4.33 \mathrm{E}-07$ & $4.90 \mathrm{E}-07$ & 4.70E-07 & $6.56 \mathrm{E}-07$ & $8.27 \mathrm{E}-07$ & 7.41E-07 \\
\hline 18 to 45 & $6.63 \mathrm{E}-05$ & 7.04E-05 & $6.97 \mathrm{E}-05$ & $1.15 \mathrm{E}-07$ & $1.28 \mathrm{E}-07$ & $1.21 \mathrm{E}-07$ & $1.71 \mathrm{E}-07$ & $1.90 \mathrm{E}-07$ & $1.80 \mathrm{E}-07$ \\
\hline 45 to 60 & $6.89 \mathrm{E}-05$ & $6.51 \mathrm{E}-05$ & $6.85 \mathrm{E}-05$ & $1.11 \mathrm{E}-07$ & $1.14 \mathrm{E}-07$ & $1.15 \mathrm{E}-07$ & $1.62 \mathrm{E}-07$ & $1.71 \mathrm{E}-07$ & $1.71 \mathrm{E}-07$ \\
\hline 60 to 80 & $6.71 \mathrm{E}-05$ & $6.21 \mathrm{E}-05$ & $6.52 \mathrm{E}-05$ & $1.04 \mathrm{E}-07$ & $1.12 \mathrm{E}-07$ & $1.10 \mathrm{E}-07$ & $1.43 \mathrm{E}-07$ & $1.52 \mathrm{E}-07$ & 1.43E-07 \\
\hline$\geq 80$ & $6.76 \mathrm{E}-05$ & $6.16 \mathrm{E}-05$ & $6.61 \mathrm{E}-05$ & $1.20 \mathrm{E}-07$ & $1.15 \mathrm{E}-07$ & $1.17 \mathrm{E}-07$ & $1.71 \mathrm{E}-07$ & $1.43 \mathrm{E}-07$ & $1.52 \mathrm{E}-07$ \\
\hline
\end{tabular}



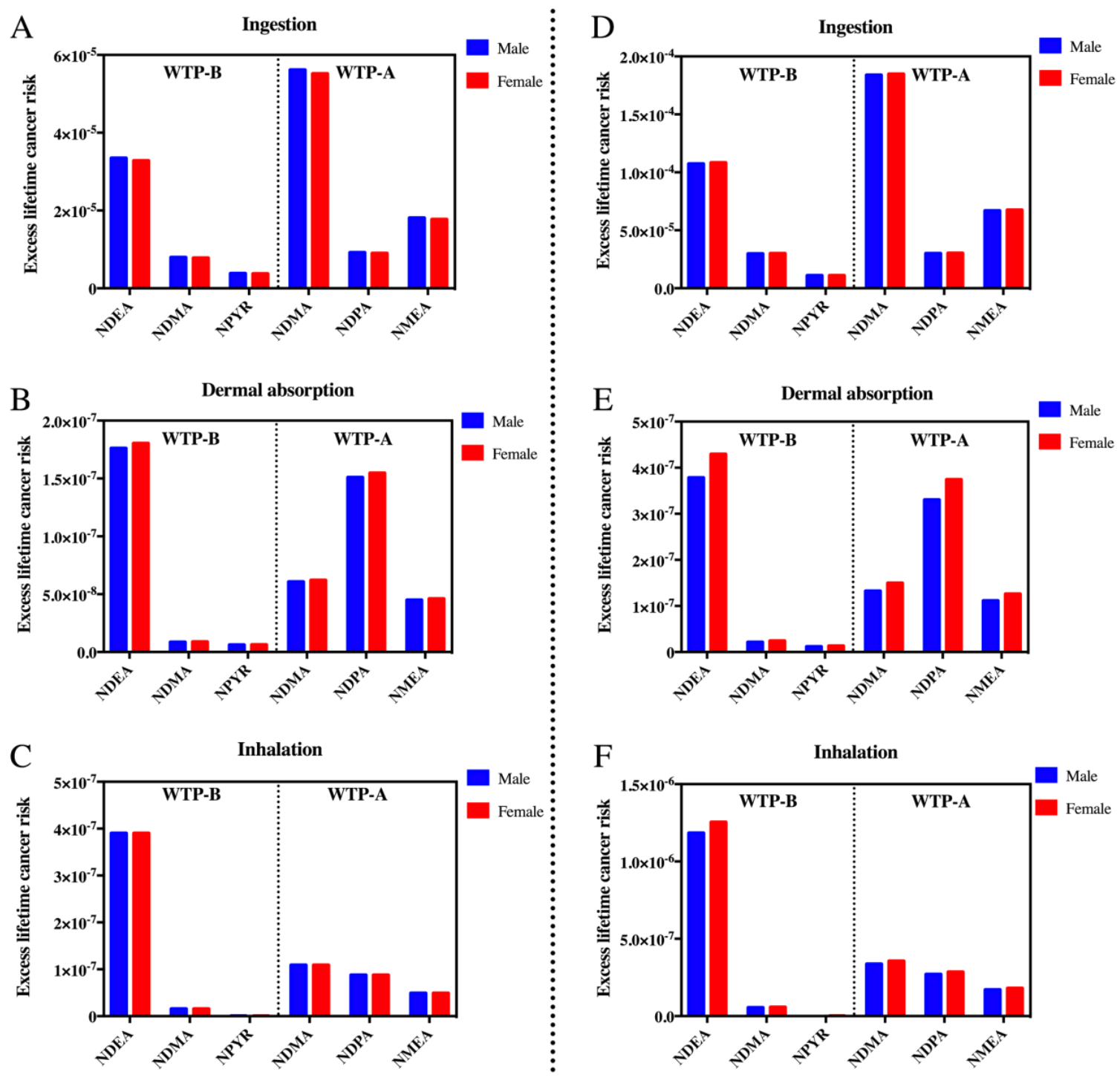

CTE scenario

RME scenario

Figure S1. Estimated lifetime cancer risk associated with ingestion, dermal contact, and inhalation of nitrosamines-contained water from WTP-A and WTP-B under different genders in CTE and RME scenarios. 
A

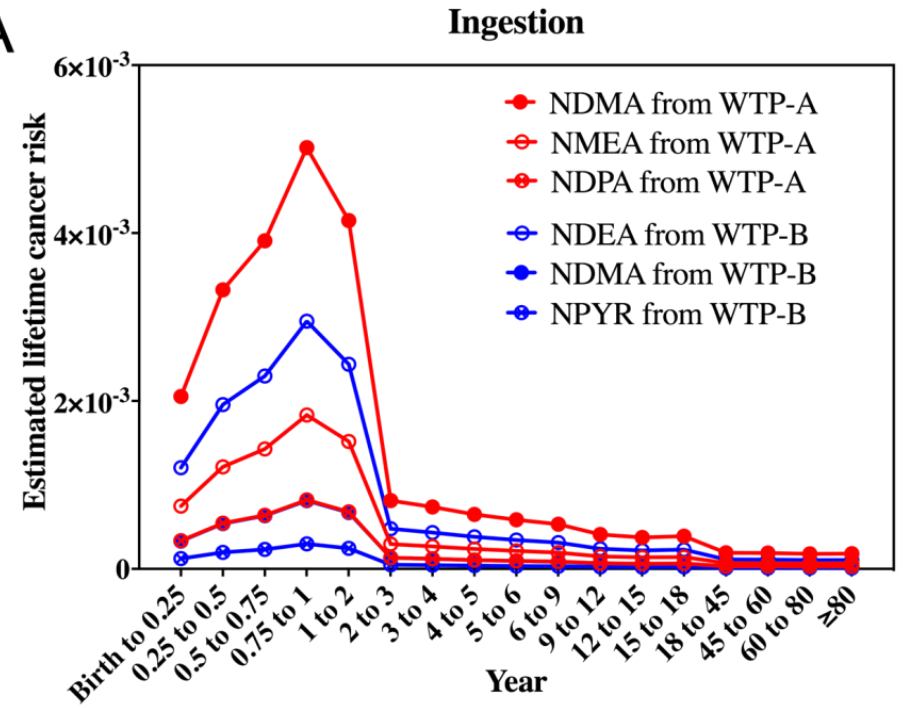

B

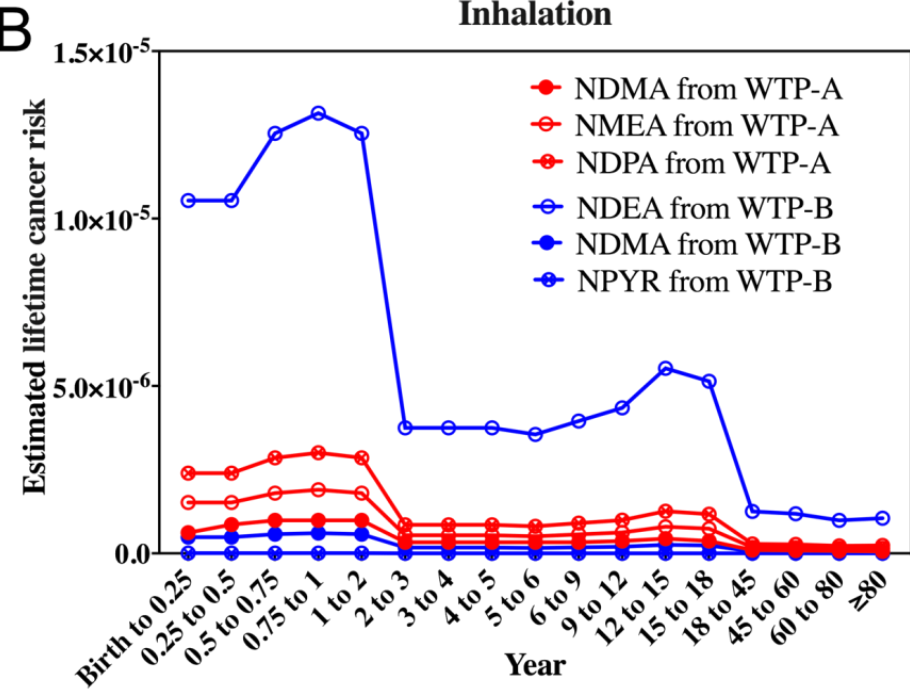

C

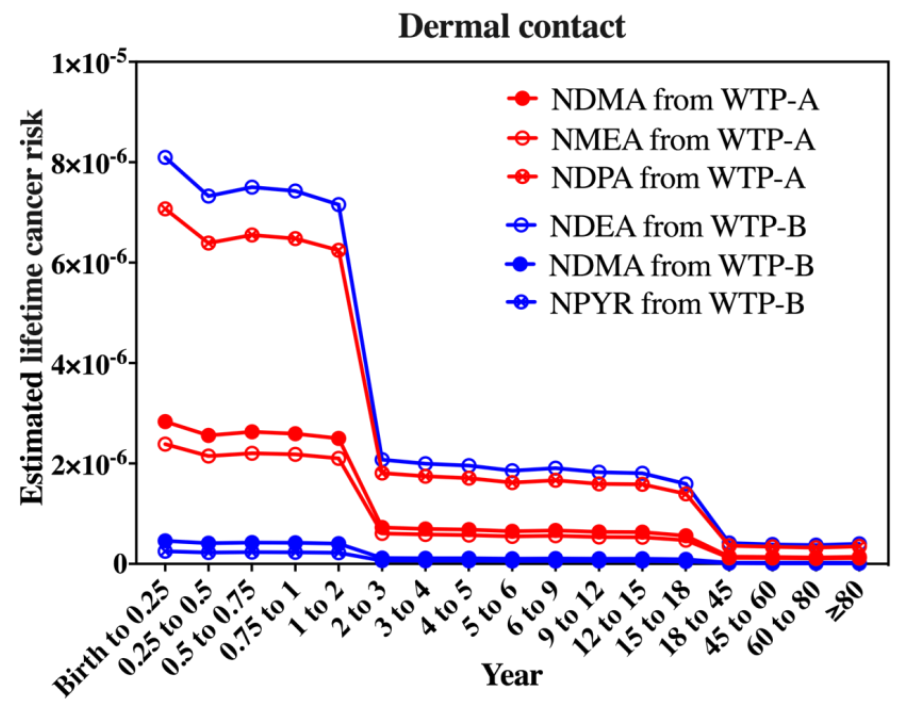

D

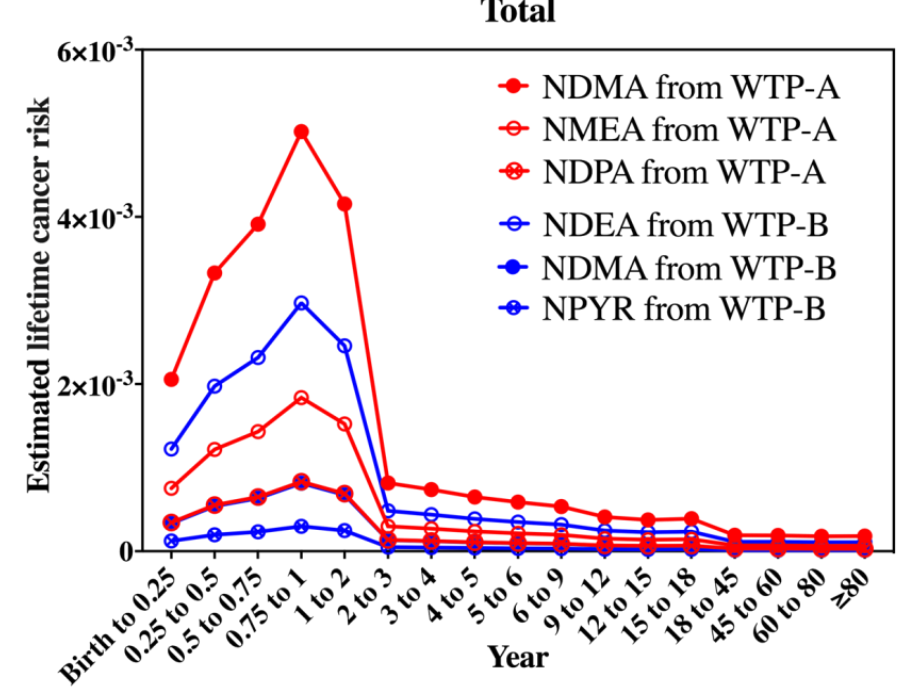

Figure S2. Estimated lifetime cancer risk associated with ingestion, dermal contact, inhalation, and total exposure of pattern of nitrosamines-contained drinking water from WTP-A and WTP-B by age groups. The risk in this figure is estimated under the reasonable maximum exposure scenario. 
Pre-oxidation with

sodium hypochlorite

Chlorination

WTP-A

Raw water $\downarrow$ Coagulation $\rightarrow$ Sedimentation $\rightarrow$ Sand filtration $\rightarrow \begin{gathered}\text { Biological activated } \\ \text { carbon filtration }\end{gathered}$

Pre-oxidation with ozone

Chlorination

WTP-B

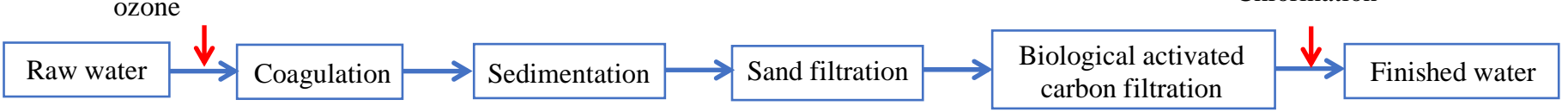

Figure S3. Treatment scheme of two water treatment plants (WTP-A and WTP-B) in Shanghai. 


\section{References}

1. Ministry of Environmental Protection. Exposure Factors Handbook of Chinese Population (0-5 years); China Environmental Science Press: Beijing, 2016.

2. Ministry of Environmental Protection. Exposure Factors Handbook of Chinese Population (6-17 years); China Environmental Science Press: Beijing, 2016.

3. Ministry of Environmental Protection. Exposure Factors Handbook of Chinese Population (adults); China Environmental Science Press: Beijing, 2013.

4. National Bureau of Statistics of China. China Statistical Yearbook; China Statistics Press: 2015.

5. U.S. EPA (Environmental Protection Agency), Exposure Factors Handbook: 2011 Edition. EPA/600/R-09/052F; Office of Research and Development: Washington, DC, 2011.

6. Murray, C. J., Quantifying the burden of disease: the technical basis for disability-adjusted life years. Bull. World. Health. Organ. 1994, 72 , (3), 429.

7. Christopher, J.L., The global burden of disease: a comprehensive assessment of mortality and disability from diseases injuries and risk factors in 1990 and projected to 2020: Summary. Harvard University Press: Boston 1996, pp 1308-1314.

8. Lopez, A. D.; Mathers, C. D.; Ezzati, M.; Jamison, D. T.; Murray, C. J., Global burden of disease and risk factors. Washington D.C., 2006, 22 , (3), $277-283$.

9. Lopez; Alan, D., Global health statistics. Harvard University Press: Boston 1996, pp 523-7.

10. U.S. EPA (Environmental Protection Agency). Regional Removal Management Level (RML) Resident Tapwater Table. https://semspub.epa.gov/work/HQ/197278.pdf (accessed March 976 18, 2019). 